\title{
7 Die Finanzkrise und ihre Folgen: Manifestationen und Repräsentationen der Krise
}

\subsection{Auswirkungen der Krise auf die psychische Gesundheit - eine exemplarische Darstellung}

„Da sich Produktions- und Machtverhältnisse ständig verändern, von Menschen gemacht und von ihnen im Prinzip veränderbar sind, dürfen sie nicht als unveränderbar beschrieben werden " (Parin, 1975, S. 524).

Die WHO (2011) hat darauf hingewiesen, dass die Gefahr von psychischen Krankheiten infolge der Wirtschaftskrise, die in vielen europäischen Ländern mit einem Anstieg der Arbeitslosigkeit, einer Zunahme der in Armut lebenden Menschen und Kürzungen von staatlichen Ausgaben einherging, steigt. Von einem wirtschaftlichen Standpunkt aus gesehen, führt eine erhöhte Zahl an Erkrankungen volkswirtschaftlich zu Produktionsausfällen, die auf durchschnittlich drei bis vier Prozent des Bruttosozialprodukts in der Europäischen Union geschätzt werden (vgl. World Health Organization, 2011, S. 1).

Im Bericht der WHO wird darauf verwiesen, dass bestimmte Maßnahmen, die von den Regierungen gesetzt werden, das Ausmaß der Schäden für die Bevölkerung und die Volkswirtschaft mildern können; dazu wird am Ende des Kapitels mehr zu lesen sein.

Überblickt man die große Menge an Forschungsdaten zu den Auswirkungen der Wirtschaftskrise in den europäischen Ländern, so stößt man bei der Komplexität der Wirkungszusammenhänge ${ }^{104}$ auf Risikofaktoren, die in den Fokus der Analyse gerückt werden: Dazu gehören die Arbeitslosenrate, Verarmung, prekäre Arbeitsverhältnisse, fehlende soziale Eingebundenheit und die Mortalitätsrate, im Speziellen die Suizidrate und der Alkohol- und Drogenkonsum (vgl. Martin-

104 "Firstly, in a complex phenomenon like this, quality study designs such as RCTs are very difficult to conceive and implement. Similarly, this complexity means that with even the most stringent analyses, assigning causality to association is challenging. Also, heterogeneity of outcomes among studies is an expected methodological hurdle in such a multifaceted issue" (Christodoulou, 2017, S. 91).

Van Hal spricht von mehreren methodologischen Problemen; das erste besteht darin, den Beginn der Finanzkrise zu definieren, das zweite ist, Maßstäbe für die psychische Gesundheit festzulegen (vgl. Van Hal, 2015, S. 18 f.). 
Carrasco et al., 2016, S. 89). $\mathrm{Zu}$ den materiellen und psychischen Langzeitwirkungen zählen auch transgenerationale Einflüsse, die aufgrund von Verarmung, Überschuldung, Wohnungsverlust, fehlenden Bildungs- und Beschäftigungsmöglichkeiten, Jugendarbeitslosigkeit und psychischen Erkrankungen erst in der Zukunft zum Tragen kommen werden. ${ }^{105}$ Sie beeinflussen politische Einstellungen und die nationale Identitätsbildung, die mit den Instrumenten der Psychoanalyse und Gruppenanalyse untersucht werden können.

Im Folgenden soll ein Überblick über die wesentlichen Forschungsergebnisse gegeben werden. Länder wie Griechenland, Spanien oder Italien waren durch die Staatsschuldenkrise und ihre Handhabung im europäischen Kontext stärker als ihre Nachbarländer von den Krisenfolgen betroffen und wurden dadurch zu einem lebendigen Laboratorium der Wissenschaftler, in dem die Auswirkungen der Wirtschaftskrise untersucht werden können. Griechenland und Italien haben darüber hinaus unter der Flüchtlingskrise zu leiden, die die Versorgungslage verschärft. ${ }^{106}$ Exemplarisch soll der Blick auf die aufgezählten Länder gelegt und sollen Gemeinsamkeiten herausgearbeitet werden.

\subsubsection{Arbeitslosigkeit/Jugendarbeitslosigkeit}

Arbeitslose gehören zu den Hauptrisikogruppen, psychisch zu erkranken. "Unemployment is confirmed as a strong association to poor mental health, in accordance with international literature" (Christodoulou, 2017, S. 91). Die europäischen Länder hatten alle unter einem Anstieg von Arbeitslosen zu leiden. ${ }^{107}$

In Spanien kam es zu einem rasanten Zuwachs der Arbeitslosigkeit, wovon besonders junge Menschen betroffen waren:

"In particular, unemployment in the population younger than 25 years increased from $19.6 \%$ in 2005 to $55.5 \%$ in 2013 [...] Since the beginning of the recession, over half a million Spanish citizens have migrated to other European countries: most of them are young, and many have a university degree" (Moreno-Küstner \& Gutierrez, 2017, S. 96).

105 "Many of the adverse effects on mental health can be pervasive or manifest fully long after the onset of the crisis. Therefore, it is possible that we are not yet able to understand the full impact of the crisis on the mental health of the European citizens" (Martin-Carrasco et al., 2016, S. 90).

106 "The conjuncture of this major financial disaster and the concurrent refugee crisis is very worrying, as the already impoverished systems of mental and social care are not coping under the pressure of austerity and demand" (Christodoulou, 2017, S. 92).

107 Siehe Abbildung 2 (Arbeitslosigkeit - Vergleich ausgewählter EU-Mitgliedstaaten seit 2008 und Stand 2016) auf Seite 19. 
In Italien nahm die Jugendarbeitslosigkeit zwischen 2009 und 2013 ebenso stark zu. "The increase in youth unemployment rate was $57.5 \%$ (from $25.4 \%$ to 40 \%)" (Ruggeri \& Tomassi, 2017, S. 104).

Psychische Erkrankungen und Arbeitslosigkeit bedingen sich teilweise wechselseitig: "A study in 27 European countries found that people with mental health problems not caused by the economic crisis are at greater risk of losing their job during economic crisis, especially men and individuals with lower levels of education" (Van Hal, 2015, S. 21).

Forschungsergebnisse aus Spanien untermauern die Annahme, dass die Effekte von Arbeitslosigkeit auf die psychische Gesundheit nicht für alle gleich sind. "Qualifiers such as being male, of working age, unemployed, with multiple economic risk and immigrant status are related to poorer mental health after the advent of the economic crisis in Spain" (Moreno-Küstner \& Gutierrez, 2017, S. 100).

Allerdings ist der Zusammenhang von Arbeitslosigkeit und Suizidalität ${ }^{108}$, der immer wieder hergestellt wird, kritisch zu betrachten. ${ }^{109}$ Wie sich zeigt, spielen hier auch andere Faktoren eine Rolle; ein Suizid ist ja oft Ausdruck einer psychischen Erkrankung bzw. der Endpunkt einer Krise. "[...] Baumbach et al found that the effect of unemployment and suicide is higher in countries with lower social spending, and Sir Michael Marmot said countries that had the greatest social spending had the lowest mortality rates" (Van Hal, 2015, S. 22 f.).

Die in vielen Staaten implementierten Sparprogramme führten auch zu Einsparungen im Gesundheitsbereich, was gerade für die Schwächsten in der Gesellschaft, die Arbeitslosen, Nicht-Versicherten oder Menschen mit geringem Einkommen - jene also, die besonders gefährdet sind, psychisch krank zu werden oder sich das Leben zu nehmen -, fatal war. "Just when people have the highest need for mental help, cost-cutting measures in the healthcare sector lead to a (substantial) drop in the supply of services for the prevention, early detection, and cure of mental health problems" (Van Hal, 2015, S. 17). In Griechenland waren steigender Personalmangel in den Gesundheitseinrichtungen, überaus lange Wartezeiten, Bestechungen oder weniger Zuweisungen die Folge

108 "Several studies have shown that unemployment is associated with suicidal thoughts" (Van Hal, 2015, S. 17).

109 "There are several factors which mediate this association, particularly the presence of psychiatric disorders, both previous and related to the hardship" (Martin-Carrasco et al., 2016, S. 104 f.).

„Moreover, Reeves et al clearly stated that unemployment is only one of the factors that increase suicide in a recession, with others, such as the rise in personal debt and mortgage foreclosures (which occur before rises in unemployment), also affecting mental health" (Van Hal, 2015, S. 21). 
(vgl. Kentikelenis et al., 2011, S. 1457). Das könnte auch den Effekt erklären, dass Menschen, die medizinische Betreuung nötig hatten, dennoch nicht zum Arzt gingen. ${ }^{110}$

"[...] during the first years of the crisis, governmental funding for mental health services was severely restricted, resulting in bed occupancies over $120 \%$, mental health professionals' wages reduced by up to $40 \%$, and research funding falling sharply, among others" (Christodoulou, 2017, S. 92).

Damit wurde die psychiatrische Versorgung massiv geschwächt (vgl. Christodoulou, 2017, S. 92), Kürzungen gab es auch im Bereich des Gesundheitsservices für Kinder und Jugendliche. "Child and Adolescent services have struggled following a simultaneous $40 \%$ reduction in healthcare workers' salaries and an escalated number of new referrals to services" (Christodoulou, 2017, S. 93).

In Spanien betrafen die Sparmaßnahmen ebenso den Bildungs- und Gesundheitssektor, was zu einem beschränkten Zugang zu gesundheitlichen Leistungen für arme Leute bedeutete. "More specifically, in 2012, a new Spanish law limited health coverage only to employees (except for minors below the age of 18 years) [...]" (Moreno-Küstner \& Gutierrez, 2017, S. 96).

Was Österreich betrifft, sieht man in Abbildung 6, dass auch hier das Wachstum der Gesundheitsausgaben nach dem Beginn der Krise stark zurückgegangen ist. „Mit einem Anstieg von real $3 \%$ hat das Wachstum im Jahr 2012 allerdings wieder zugelegt" (OECD, 2014, S. 1). In Österreich werden 76 Prozent der Gesundheitsausgaben von der öffentlichen Hand finanziert (vgl. OECD, 2014, S. 1).

\subsubsection{Suizide/Mortalität}

In Griechenland stiegen die Selbstmorde von 2007 bis 2009 um 17 Prozent (Kentikelenis et al., 2011, S. 1457) und im Vergleich dazu 2010 bis zur ersten Hälfte 2011 um 40 Prozent (Van Hal, 2015, S. 19). "The national suicide helpline reported that $25 \%$ of callers faced financial difficulties in 2010 and reports in the media indicate that the inability to repay high levels of personal debt might be a key factor in the increase in suicides" (Van Hal, 2015, S. 21).

110 "In Greece, despite their feeling it was necessary, there was a rise of $15 \%$ of people not going to the doctor and $14 \%$ in people not going to the dentist" (Van Hal, 2015, S. 20). 


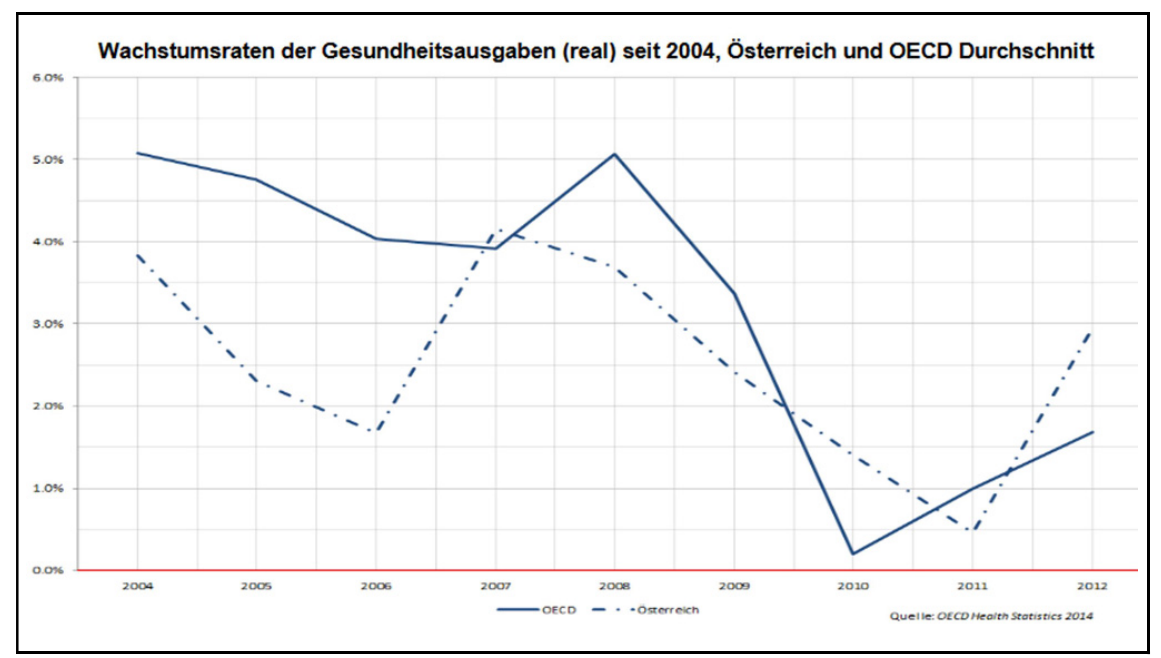

Abbildung 6: Wachstumsraten der Gesundheitsausgaben (OECD, 2014, S. 1)

Neue Studien bestätigen den Zusammenhang, wobei Männer im arbeitsfähigen Alter als Risikogruppe besonders hervortreten. Dasselbe gilt nicht für Frauen (vgl. Christodoulou, 2017, S. 91). Ebenso wird eine Zunahme an Gewalttaten beschrieben. "Violence has also risen and homicide and theft rates nearly doubled between 2007 and 2009" (Kentikelenis et al., 2011, S. 1457 f.).

Der Verlust an Arbeit wird in Italien im Jahr 2013 zum zweithäufigsten Auslöser für Selbstmorde, weitere Gründe sind Schulden (vgl. Ruggeri \& Tomassi, 2017, S. 108). Auffällig ist, dass Verkehrsunfälle abnehmen. "The decline in transport accident mortality also might be attributed to less economic activity. The fall in traffic accidents is one of the reasons that all-cause mortality rates did not show an increase during the economic crisis" (Van Hal, 2015, S. 20).

Van Hal führt eine Reihe von Forschungsergebnissen zusammen, die im Verlauf der ökonomischen Krise eine Zunahme an Selbstmorden in 26 europäischen Ländern feststellen, wenn auch mit durchaus unterschiedlichen Ergebnissen. "Countries most hit by the economic crisis, such as Greece and Ireland, had greater rises in suicides [...] than other countries" (Van Hal, 2015, S. 19). 
Österreich bildet da eine Ausnahme. ${ }^{111}$

„Seit den 1980er Jahren ist zwar ein deutlicher Rückgang der Suizidhäufigkeit zu beobachten, mit Beginn der gegenwärtig andauernden weltweiten Wirtschaftskrise im Jahr 2008 verlangsamte sich dieser Rückgang jedoch deutlich und der Wert blieb seither nahezu konstant. Der relative Rückgang der Suizide seit 1986 ist bei den Frauen deutlich stärker ausgeprägt als bei den Männern" (Grabenhofer-Eggerth, Nowotny, Tanios \& Kapusta, 2017, S. 5).

Am Schluss soll noch auf die Ausführungen von Durkheim (2014) hingewiesen werden, der Ende des 19. Jahrhunderts unter anderem den Zusammenhang zwischen wirtschaftlichen Krisen und Selbstmorden beleuchtet hat. Er belegt ihn mit Zahlen zu den Finanzkrisen im Jahr 1874 in Wien und Frankfurt bzw. mit jenen nach dem Börsenkrach 1882 in Paris (vgl. Durkheim, 2014, S. 273). Dabei sind es nach Durkheim nicht so sehr die Folgen, wie ein sinkender Lebensstandard und die damit einhergehende Verarmung, die diesen Anstieg erklären, wie sich vermuten ließe. Denn paradoxerweise tritt ein ähnlicher Effekt in Zeiten plötzlicher Konjunktursteigerung ein. „Das scheint vor allem bei der Weltausstellung von 1878 der Fall gewesen zu sein. [...] Sie [Die Selbstmordrate] betrug $8 \%$ und war damit höher als die durch den Börsenkrach von 1882 verursachte“ (Durkheim, 2014, S. 277).

Wie kann man sich das erklären? Nach Durkheim werden die Bedürfnisse und Befriedigungsmöglichkeiten der Menschen in einer Gesellschaft sozial geregelt. Es existieren ungeschriebene Gesetze, die jeder sozialen Gruppe ein bestimmtes Maß an Wohlstand und Bedürfnisbefriedigung zuerkennen. Er schreibt: „Früher war fast ausschließlich die Geburt für die Zugehörigkeit zu einer sozialen Schicht ausschlaggebend, heute gibt es eine ursprüngliche Ungleichheit nur auf Grund ererbten Vermögens oder Verdienstes“" (Durkheim, 2014, S. 285).

In Krisenzeiten jedoch kommt es zu "Störungen der kollektiven Ordnung“ (Durkheim, 2014, S. 278). Die Folge ist, dass das soziale Reglement infolge der Instabilität seine Wirkung verliert. „Der Zustand der gestörten Ordnung oder Anomie $^{112}$ wird also dadurch noch verschärft, daß die Leidenschaften zu einem Zeitpunkt, wo sie einer stärkeren Disziplin bedürfen, weniger diszipliniert sind!“ (Durkheim, 2014, S. 289). Mit dem Begriff des „anomischen Selbstmords“ be-

111 „Eine jüngst durchgeführte Studie zeigt, dass der Grund für die seit 1987 kontinuierlich sinkenden Suizidraten in Österreich nicht nur in sozialen und finanziellen Absicherungsprogrammen begründet liegt. So hat zum Beispiel die Verschärfung des Waffengesetzes zur Reduzierung der Suizidraten beigetragen“ (Berghofer \& Psota, 2013, S. 149).

112 Hervorhebung durch Durkheim. 
zeichnet Durkheim jene Fälle, bei denen Menschen aufgrund des Leidens infolge von Regellosigkeit zur Selbsttötung greifen (vgl. Durkheim, 2014, S. 296).

Nur für einen Bereich der Gesellschaft gilt dieser Zusammenhang laut Durkheim nicht - wie er in Bezug auf das 19. Jahrhundert feststellt -, nämlich für die „Welt des Handels und der Industrie“, dort ist die Regellosigkeit „eine Art Dauerzustand“ (Durkheim, 2014, S. 290). „Seit einem Jahrhundert besteht der wirtschaftliche Fortschritt hauptsächlich darin, das Gewerbe von jeder Reglementierung zu befreien“ (Durkheim, 2014, S. 291). In vergangenen Zeiten hatten Religion und Innungswesen die Aufgabe, die soziale Ordnung herzustellen und zu festigen, jedem Menschen innerhalb der Gesellschaft seinen Platz zuzuweisen (vgl. Durkheim, 2014, S. 291). Wirtschaftliches Wachstum geht jedoch mit Bedürfnissteigerung einher, ist auf Expansion und nicht auf Begrenzung gerichtet.

Durkheim äußert sich sehr kritisch gegenüber jenen Bestrebungen in der Gesellschaft, die heute im Vergleich zu damals viel stärker geworden sind, die den „Hunger nach neuen Dingen, nach unbekannten Genüssen, nach Freuden ohne Namen“ (Durkheim, 2014, S. 293) befördern. Daher ist es auch nicht überraschend, wie sich die Selbstmordrate in Berufen zeigt, die diesen Bereichen zuzuordnen sind. „Die Bereiche von Handel und Industrie zählen tatsächlich zu den Berufen mit den meisten Selbstmorden. Sie haben fast so viele wie die freien Berufe, und manchmal sogar noch mehr" (Durkheim, 2014, S. 294).

Meine Interviewpartner, besonders aus dem Bankenbereich, haben auf Suizide und Suizidgefahren hingewiesen. Aus Medienberichten erfuhr man im Anschluss an die Finanzkrise von Bankmanagern, die viel Geld verloren hatten und sich das Leben nahmen. ${ }^{113}$ Die Motive, die dazu führen, reichen von Angst vor Strafverfolgung bis hin zu Überlastung. Für Erstere trifft möglicherweise die Einschätzung von Durkheim zu: „Aber diejenigen, die über sich nur die Leere haben, verlieren sich fast mit Notwendigkeit darin, wenn sie keine Macht mehr haben“" (Durkheim, 2014, S. 295).

113 „Tatsächlich sind in der Finanzkrise mehrere Selbstmorde bekannt geworden. Barry Fox, Manager bei Bear Stearns, war der erste [...] Der bisher prominenteste reale Wall-Street-Selbstmordfall war der von Thierry de la Villehuchet. AIA, die Fondsfirma des französischen Aristokraten mit New Yorker Wahlwohnsitz, hatte fast 1,5 Milliarden Dollar in der Affäre Madoff verloren, darunter auch Villehuchets Privatvermögen. Am Abend des 23. Dezember schloss sich Villehuchet, 65, in seinem Büro im 22. Stock an der Madison Avenue in Midtown Manhattan ein, setzte sich hinter seinen Schreibtisch, nahm Schlaftabletten und schnitt sich die Pulsadern auf* (http://www.spiegel.de/wirtschaft/echte-und-falsche-krisen-selbstmorde-vermoegendverzockt-verzweifelt-a-603449.html). 


\subsubsection{Alkohol- und Drogenkonsum}

Interessanterweise nahm der Alkoholkonsum, auch das alkoholisierte Autofahren in Griechenland im Zuge der Wirtschaftskrise, ab, obwohl die Kontrollen im Straßenverkehr gleich geblieben waren (vgl. Kentikelenis et al., 2011, S. 1458). Andere Untersuchungen bestätigen diese Ergebnisse, sehen aber eine Zunahme an „Binge Drinking“ bei Risikogruppen wie Arbeitslosen (vgl. Martin-Carrasco et al., 2016, S. 102; Van Hal, 2015, S. 20).

Bereits in den ersten Jahren nach Ausbruch der Krise kam es in Griechenland $\mathrm{zu}$ einer Zunahme an HIV-Infektionen. "The latest data suggest that new infections will rise by $52 \%$ in 2011 compared with 2010 (922 new cases versus 605) [...]" (Kentikelenis et al., 2011, S. 1458).

Die Studienautoren sehen diese Entwicklungen in Verbindung mit den Einsparungen im Bereich der Streetworker-Programme und mit einer Zunahme der Prostitution. "This is also produced by scaled-back preventive programs, for example, needle exchange and early treatment services [...]" (Van Hal, 2015, S. 20).

"In general, people facing financial difficulties are at greater risk for mental disorders. In Britain, people mired in debt have two to three times the frequency of depression or psychosis, double that of alcohol dependence, and four times that of drug dependence compared with the general population" (Van Hal, 2015, S. 21).

Psychische Erkrankungen, die mit wirtschaftlichen Krisen einhergehen bzw. ihnen folgen, sind affektive Störungen, Angsterkrankungen und alkoholbezogene Erkrankungen. In Griechenland hat sich die Prävalenzrate für die Major Depression zwischen 2008 und 2011 verdoppelt (vgl. Van Hal, 2015, S. 19). "Based on the comparison of two telephone surveys conducted in 2008 and 2011, the odds of having major depression increase were 2.6 times higher in 2011 compared to 2008, with economic hardship being a significant association" (Christodoulou, 2017, S. 91).

In Spanien nahmen die affektiven Störungen, Angststörungen und alkoholbezogenen Störungen zwischen 2006 und 2010 zu, ebenso Fälle von Major Depression in Zusammenhang mit Problemen bei Darlehensrückzahlungen und Zwangsräumungen (vgl. Van Hal, 2015, S. 19 f.). "This results show a sharp increase in mood disorders $(30.2 \%)$, anxiety disorders $(14.4 \%)$, somatoform disorder $(7.2 \%)$ and alcohol abuse and dependence $(7 \%)$ during the crisis" (Martin-Carrasco et al., 2016, S. 93).

"A significant psychotropic drug consumption in Greece was found in the period following the economic crisis" (Moreno-Küstner \& Gutierrez, 2017, S. 97). "A study performed between 2000 and 2011 using the Italian Medicines Agency database, proved that the increase of antidepressant consumption has drastically 
increased, between 2000 and 2011, from 8.18 to 36.12 Defined Daily Dose (DDD), with a higher increase for selective serotonin uptake inhibitors" (Ruggeri \& Tomassi, 2017, S. 107).

Die OECD stellt ebenso einen möglichen Zusammenhang zwischen der Zunahme des Antidepressivakonsums und den Krisenfolgen her.

"Some of the increases in the use of antidepressants may also be linked to the insecurity created by the economic crisis (Gili et al., 2012). In Spain, the consumption of antidepressants per capita has increased by $23 \%$ between 2007 and 2011, although this increase was slightly lower than in the preceding four-year period (28\% between 2003 and 2007). In Portugal, antidepressant consumption went up by $20 \%$ between 2007 and 2011. The consumption of antidepressants rose even more quickly in countries such as Germany (a rise of 46\% between 2007 and 2011) which were less affected by the economic crisis and have experienced a more rapid economic recovery" (OECD, 2013, S. 102).

In den Abbildungen 7 und 8 aus den „Health at a Glance“-Berichten der OECD sieht man, wie sich der Konsum von Antidepressiva in den beschriebenen Zeiträumen verändert hat.

Riggeri und Tomassi formulieren vorsichtig: "Use of AD [antidepressants] seems to be a sensitive marker connected to social changes and also to the economic crisis" (Ruggeri \& Tomassi, 2017, S. 107).

Schlafprobleme werden als weit verbreitet beschrieben und mit nachfolgenden psychischen oder physischen Gesundheitsproblemen in Verbindung gebracht. "Therefore, it is not surprising that several studies have explored specifically the relationship between economic crisis and sleep disturbances" (MartinCarrasco et al., 2016, S. 98).

Es besteht unter den vorliegenden Studien eine große Übereinstimmung, dass Sparprogramme im Gesundheits- und Sozialwesen negative Effekte für die Menschen ebenso wie für die Wirtschaft insgesamt haben. ${ }^{114}$

"Several examples show that the countries best off regarding the mental health of their populations during the economic crisis are those countries with the strongest social safety net. Therefore, instead of cutting back on healthcare and social welfare measures, policymakers should in the future invest even more in social protection measures during economic crisis" (Van Hal, 2015, S. 17).

114 Auch wenn Van Hal behauptet, "Iceland rejected austerity through a popular vote, and the financial crisis seems to have had few or no discernible effect on health in the country" (2015, S. 22), lässt die Entwicklung des Antidepressivakonsums (Abbildung 8) Zweifel an dieser Aussage aufkommen. 


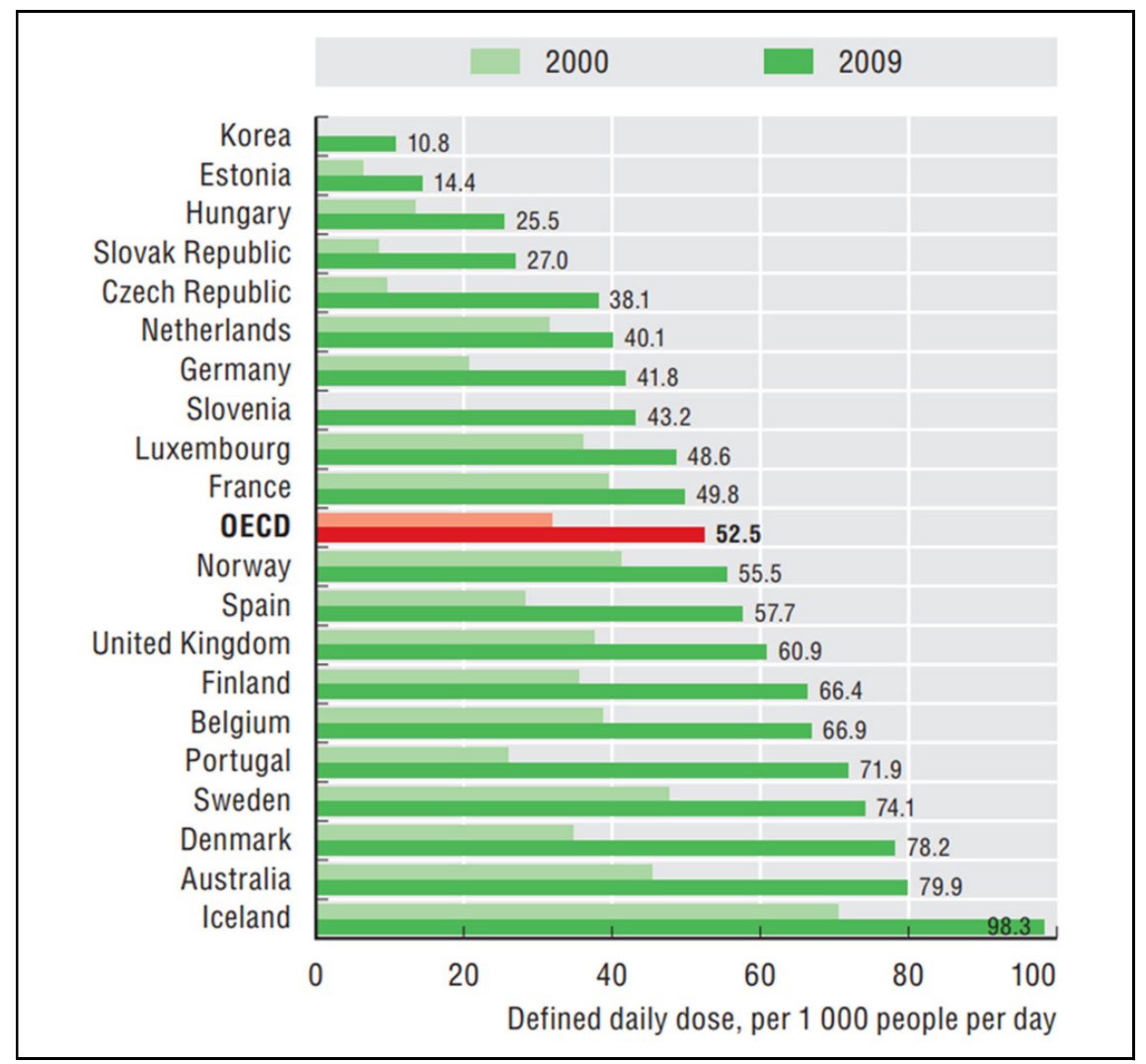

Abbildung 7: $\quad$ Konsum von Antidepressiva 2000 und 2009 (OECD, 2011, S. 101)

Immer wieder wird jedoch auch thematisiert, dass familiäre und soziale Bindungen sowie der Bildungsstand den Effekt der Krise teilweise abgefedert oder verzögert und sich als Resilienzfaktoren erwiesen haben. ${ }^{115}$ Ganz in diesem Sinne verweist die WHO darauf, dass Wirtschaftskrisen durch ein starkes soziales Netz abgefedert werden können, was das Risiko psychischer Erkrankungen mindert und gleichzeitig zu einer schnelleren Erholung der Wirtschaft führt (vgl. World Health Organization, 2011, S. 8). Als Schutzmaßnahmen werden von der WHO aktive Arbeitsmarktprogramme, Unterstützungsprogramme für einkommens-

115 Siehe dazu Ruggeri und Tomassi (2017, S. 109) sowie Christodoulou (2017, S. 93). 


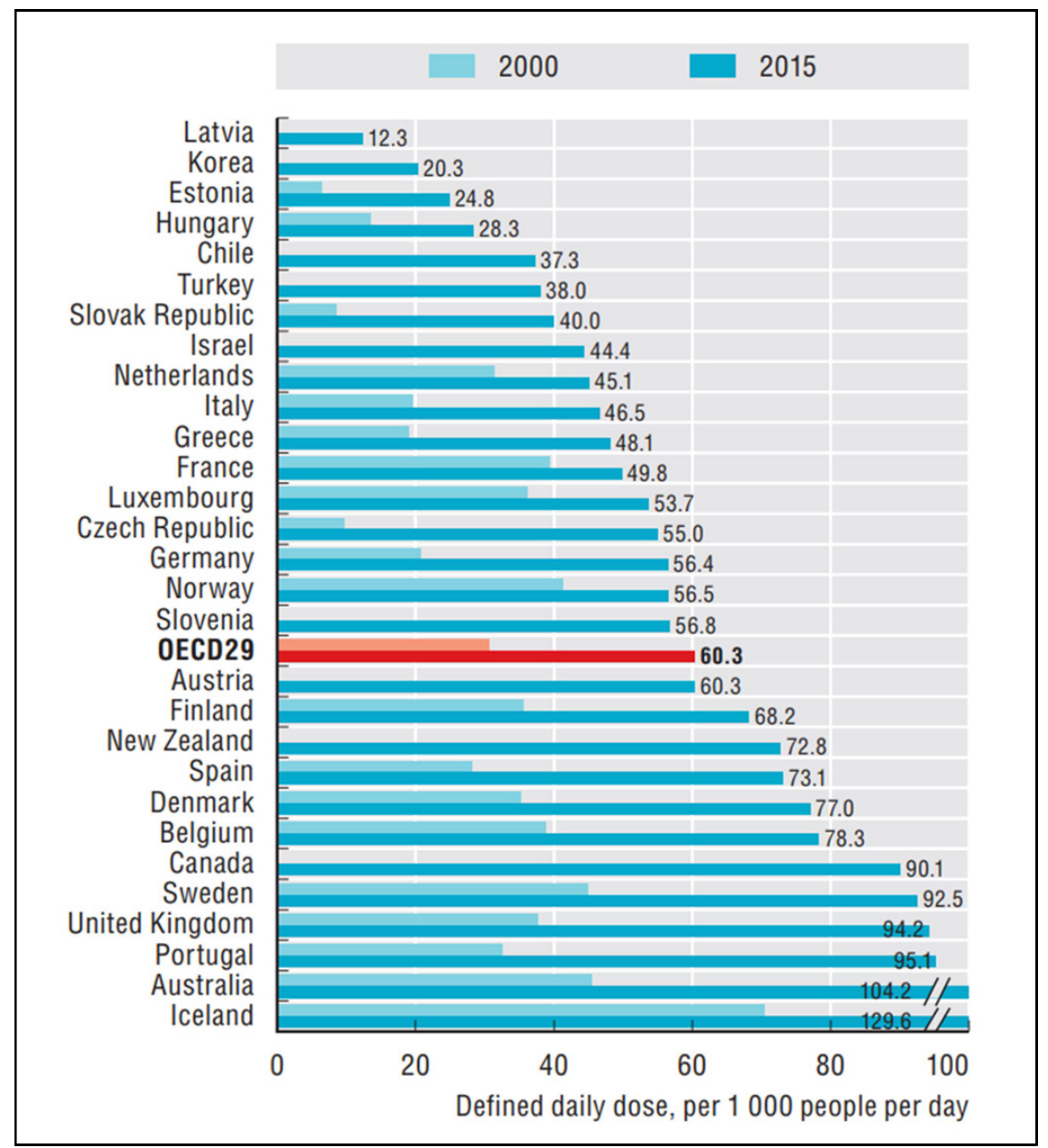

Abbildung 8: $\quad$ Konsum von Antidepressiva 2000 und 2015 (OECD, 2017, S. 191)

schwache Familien, eine Kontrolle der Alkoholpreise und der Verfügbarkeit, Primärversorgung für Menschen, die ein hohes Risiko haben, psychisch zu erkranken, und Schuldennachlassprogramme vorgeschlagen (vgl. World Health Organization, 2011, S. 10 f.).

Am Ende dieses Kapitels möchte ich auf eine andere, nicht mehr existente Kultur verweisen, in der das Wissen um diesen Zusammenhang zwischen ökonomischen Verhältnissen und psychischem Wohlergehen zu bestimmten sozialen 
Praktiken geführt hatte, die Wünschen zum Ausdruck verhalfen und gleichzeitig materiell und sozial ausgleichende Wirkung hatten. ${ }^{116}$ Ellenberger beschreibt, auf Berichte von Jesuiten aus dem 17. Jahrhundert Bezug nehmend, die krankmachende Wirkung von unerfüllten Wünschen, die sich in den Träumen von Indianern im Nordosten Amerikas zeigten. Die Heilung erfolgte im Rahmen von Festen, in denen zugunsten der Erkrankten Kollekten durchgeführt wurden:

„So pflegte der Patient nicht nur von seiner Krankheit zu genesen und alle seine Wünsche erfüllt zu bekommen, sondern er wurde oft auch ein reicher Mann. Andererseits wurden einige der Geber nun vielleicht ihrerseits krank und träumten davon, daß sie eine Entschädigung für ihren Verlust bekämen" (Ellenberger, 2005, S. 55).

Man kann sich vorstellen, dass sich die ökonomischen Verhältnisse innerhalb dieser Gemeinschaft ständig verschoben haben.

In der Erforschung und Behandlung von psychischen Erkrankungen wird oftmals die soziale und ökonomische Komponente außer Acht gelassen oder in den Hintergrund gedrängt. Angesichts des Effekts der Finanzkrise war dies nicht mehr möglich. Die vorangegangene Darstellung bestätigt, dass das psychische Wohlergehen entscheidend von externen Faktoren beeinflusst wird. Darauf wird sowohl im empirischen Teil meiner Arbeit Bezug genommen werden als auch im nächsten Kapitel, das sich mit dem Resilienzdiskurs und Subjektivierungstendenzen in den Sozialwissenschaften und in der Psychologie auseinandersetzt.

\subsection{Der Resilienz- und Optimierungsdiskurs - das Individuum als Schauplatz der Krise}

In der Bewertung eines Krisengeschehens, wie es die Finanz- und Wirtschaftskrise darstellt, und seiner Folgen gerät man unweigerlich in das Spannungsfeld zwischen Individuum und Gesellschaft. Das betrifft nicht nur die Ursachen der Krise, sondern auch den Umgang mit den Folgen. Die Ursachen können in individuellen Strebungen wie der Gier oder den moralischen Verfehlungen einzelner Menschen festgemacht werden oder in den Charakteristika des kapitalistischen Wirtschaftssystems, welches im Zuge von Globalisierung, Digitalisierung und Automatisierung neue Blüten treibt und ganze Gesellschaften und Kulturen verändert. Dasselbe gilt natürlich auch für die Folgen von Krisen und deren Bewältigung. Geht es um eine Steigerung der Widerstandsfähigkeit der Individuen oder um eine Veränderung im Umgang mit Ressourcen, zu denen auch der Mensch

116 Ich verdanke diesen Hinweis Frau Mag. Sabine Schlüter. 
als Teil eines Wirtschaftssystems gehört? Die Trennung ist eine künstliche, die noch dazu das Risiko der Einseitigkeit und Parteilichkeit birgt. Im gegebenen Fall ist sie jedoch notwendig, um den Blick zu schärfen und eben diese Gefahren zu umschiffen.

\subsubsection{Das Modell der Resilienz}

"Greece is demonstrating remarkable resilience. For example, despite the devastating crisis, it serves as an example of ethos, currently offering refuge and solidarity to a vastly disproportionate number of refugees for its size. [...] Similarly, solidarity and support to other vulnerable groups (e.g. the elderly, the mentally ill, the poor and unemployed) can serve to enhance collective resilience further, and should be advocated for as part of the solution to the crisis" (Christodoulou, 2017, S. 93).

Der Duden definiert Resilienz als „psychische Widerstandskraft“, als „Fähigkeit, schwierige Lebenssituationen ohne anhaltende Beeinträchtigung $\mathrm{zu}$ überstehen““ ${ }^{117}$ Lateinisch „resilire“ bedeutet so viel wie „zurückspringen“.

Überblickt man den Wikipedia-Eintrag zu Resilienz ${ }^{118}$, merkt man, dass der Begriff mittlerweile in verschiedene Wissenschaftsbereiche Eingang gefunden hat, wobei er ursprünglich den technischen Disziplinen der Material- und Ingenieurwissenschaften zugeordnet worden war. Zwei Charakteristika stehen im Vordergrund, nämlich die „Fähigkeit von technischen Systemen, bei einem Teilausfall nicht vollständig zu versagen“ und die „Fähigkeit eines Materials, nach einer elastischen Verformung in den Ausgangszustand zurückzukehren“. ${ }^{119}$

Wird der Begriff auf soziale Systeme übertragen, beschreibt er die Fähigkeit von Menschen und Gemeinschaften, Krisen oder Katastrophen aufgrund bestimmter Merkmale besser zu überstehen als andere. Wie Von Freyberg ausführt, war das Resilienzkonzept „ursprünglich eng mit der Traumaforschung verbunden“" (Freyberg, 2017, S. 24).

Dass ein ursprünglich ressourcenorientiertes Modell im Laufe der letzten Jahre zunehmend infrage gestellt wird, hat nicht nur mit der Ambivalenz des Begriffes und seines Bedeutungshorizonts $\mathrm{zu}$ tun, wie im Weiteren genauer beschrieben wird, sondern auch mit der Verwendung des Konzepts in unterschiedlichen Kontexten.

„Resilienz ereignet sich nicht in einem Vakuum, sondern sie greift auf vergangene Erfahrungen zurück: auf das kollektive Gedächtnis und auf die Sozialgeschichte und sie ist gleichermaßen von solchen kritischen Momenten abhängig, in denen sich der

117 http://www.duden.de/rechtschreibung/Resilienz, 9.8.2017.

$118 \mathrm{Vgl}$. https://de.wikipedia.org/wiki/Resilienz, 8.9.2017.

119 https://de.wikipedia.org/wiki/Resilienz, 8.9.2017. 
Machtapparat für oder gegen das Handeln entscheidet. Die Bedeutungen der Schocks und der kritischen Momente, die Resilienzstrategien hervorbringen, sind gesellschaftlich konstruiert. Endogene und exogene Schocks sind interpretative Momente [...] “ (Bourbeau, 2017, S. 91).

Bourbeau differenziert zwischen drei Modi der Resilienz: „Resilienz als Randerscheinung", „Resilienz als Erneuerung" und „Resilienz als Aufrechterhaltung“ (Bourbeau, 2017, S. 88). Während Ersterer die Grundlagen der Politik oder einer Gesellschaft nicht wirklich infrage stellt oder verändert, ist dies beim zweiten Modus sehr wohl der Fall, allerdings wieder, wie schon oben beschrieben, im Rahmen des Rückgriffs auf vergangene Erfahrungen (vgl. Bourbeau, 2017, S. 88).

Bei der dritten Variante werden eigene Werte und Vorstellungen, besonders der Zusammenhalt der Gruppe, als schützenswert betrachtet.

„Folgerichtig wird der Diskurs die negativen, die Gesellschaft verändernden Folgen hervorheben, die diese Schocks potenziell mit sich bringen könnten. Diese nach innen gerichtete Strategie bietet davon überzeugten Akteuren die Gelegenheit, eine neu auftretende Störung als eine Bedrohung für die Sicherheit darzustellen, was wiederum eine harte und schnelle Reaktion erfordert" (Bourbeau, 2017, S. 89).

Damit geht das Resilienzkonzept eine Allianz mit dem Sicherheitskonzept ${ }^{120}$ ein.

„So wird im Kontext der internationalen Migration eine Gesellschaft, die sich für den Modus der Resilienz als Aufrechterhaltung entscheidet, jegliche rhetorische und diskursive Macht aufbieten, um internationale Migrationsbewegungen als einen bedeutenden Schock für die Sicherheit im eigenen Land und als Bedrohung für die kollektive Identität zu deuten " (Bourbeau, 2017, S. 90).

In einer kritischen Auseinandersetzung mit dem Resilienzkonzept fallen drei Bereiche auf, die im Folgenden diskutiert werden: Resilienz als Geschäftsmodell, Resilienz als Anpassungsleistung und Resilienz als Teil eines Sicherheitskonzeptes, in dem die Zukunft nur negativ gedacht wird.

120 Ähnlich argumentiert auch Bauman, der das Konzept der „Versicherheitlichung“ als „Taschenspielertrick“ bezeichnet, der dazu diene, die Angst vor Problemen, die der Staat nicht zu lösen vermag, zu verschieben (vgl. Bauman, 2017a, S. 33 f.).

Die „Folgen des gegenwärtigen Hangs zur Versicherheitlichung des ,Migrationsproblems' und der Aufnahme oder Abweisung von Flüchtlingen und Asylsuchenden $[\ldots]$ werden $[\ldots]$ sogar noch dadurch übertroffen, dass immer mehr Regierungen ganz offiziell die im Volk verbreitete ,Sicherheitspanik' schüren [...]" (Bauman, 2017a, S. 45 f.). 


\subsubsection{Resilienz als neoliberales Geschäftsmodell}

„,Resilienz' ist zum neuen Fetisch des liberalen Staates geworden" (Neocleous, 2017, S. 151).

Die Vereinnahmung des Resilienzmodells durch Vertreter von ökonomischideologischen Konzepten wie dem Neoliberalismus passt sehr gut zu deren Auffassung, dass der Staat sich aus bestimmten Bereichen zurückziehen und die Verantwortung dem Einzelnen übertragen soll.

„Ein komplett neues Staatsverständnis kann sich unter solchen Umständen breitmachen. Eines, das die Verantwortung für die Bewältigung von Armut, die Folgen des Klimawandels und die Auswirkungen der in der Welt grassierenden Gewalt an [sic!] Subsysteme wie Familien, Kommunen, Nachbarschaften, TherapeutInnen, PädagogInnen, Hilfswerken, Unternehmen und schließlich an jede Einzelne und jeden Einzelnen abwälzt. Und so entpuppt sich Resilienz - wie ,Charity - als Teil jener neoliberalen Hegemonie [...] “ (Gebauer, 2017, S.17).

Es werden Aufgaben und finanzielle Leistungen neu verteilt, das heißt Strategien im Management von Krisen verändert, die, so die Kritiker, Ungleichheiten nicht beseitigen, sondern verstärken.

„,Resilienzförderung bedeutet in der Praxis, die Idee der Nachhaltigkeit aufzugeben und Mittel auf Kosten der Bedürftigen zu kürzen. Resilienz wird auch zum Schlüsselwort für einen grundlegenden Paradigmenwechsel auf der systemischen und der Finanzierungsebene. Unter diesem Stichwort wird das Zusammenlegen von Budgets und Strukturen diskutiert" (Merk, 2017, S. 140).

Resilienz ist mittlerweile auch zu einem lukrativen Geschäftsfeld geworden. So hat die Präsidentin der Rockefeller Foundation, Judith Rodin, 2015 ein Buch mit dem Titel „The Resilience Dividend“ verfasst, wo sie diese Gedanken ausführt und große Resonanz bei multinationalen Konzernen gefunden hat (vgl. Merk, 2017, S. 133 f.).

Resilienz wird auch zunehmend als Mess- und Kontrollinstrument für Fördergelder eingesetzt:

„Die ,Depth of Poverty'-Messung bestimmt, wie viel Armut resilient auszuhalten ist; das ,Moderate to Severe Hunger'-Instrument definiert, ab wann man wirklich verhungert, die ,Global Acute Malnutration'-Skala misst, wann die Unterernährung noch unter das übliche Maß fällt" (Merk, 2017, S. 245).

Der Fokus verschiebt sich immer mehr weg von den Ursachen und hin zu den Folgen. Es werden auch Messinstrumente erstellt, die entscheidend dazu beitragen sollen, bestimmte Risikogruppen und Regionen auszusieben (vgl. Merk, 2017, S. 130). Es geht „immer mehr um das Management von Katastrophen und 
die Anpassung an katastrophenbedingte Veränderungen [...]“ (Merk, 2017, S. 131).

Ob dies aus politischem Kalkül oder in einer Art resignativer Zustimmung zu der Unveränderlichkeit von gefährdenden Einflüssen erfolgt, bleibt offen. Es erweckt jedenfalls den Eindruck, dass Katastrophen und Krisen lokal begrenzt gehalten werden können, auch wenn die Ursachen als solche sich nicht auf diese Weise eingrenzen lassen. Das Ungleichgewicht zwischen ärmeren und reicheren Ländern, zwischen dem Norden und dem Süden, bleibt bestehen. Denn die meisten Naturkatastrophen und kriegerischen Auseinandersetzungen betreffen nach wie vor ärmere Länder.

„[...] von Naturkatastrophen sind zu 90 Prozent Menschen in sogenannten Entwicklungsländern betroffen. Das Risiko eines Landes, Opfer einer solchen Katastrophe $z u$ werden, [...] ist maßgeblich durch gesellschaftliche und ökonomische Faktoren geprägt" (Merk, 2017, S. 127).

Aus psychoanalytischer Sicht handelt es sich um innere und äußere Abwehrprozesse, wobei Letztere marktspezifischen und politischen Kriterien gehorchen. Dazu gehören auch die verwendeten Terminologien, die durch Verharmlosung und Beschönigung Realitäten verändern. „Der Resilienzdiskurs formuliert Verlust und Verwundbarkeit in der Sprache von Fortschritt und Transformation“ (Oels, 2017, S. 74).

\subsubsection{Resilienz als Anpassungsleistung}

Hauptkritikpunkt ist hier, dass die Anpassungsfähigkeit der Menschen nach Katastrophen oder wirtschaftlichen Krisen als Argument dafür angeführt wird, nicht mehr die Ursachen selbst bekämpfen zu müssen, sondern sie als gegeben anzuerkennen. Im Zuge dessen werden alte Ungleichheiten, wie schon weiter oben angesprochen, vertieft oder neue geschaffen.

„Der Resilienzdiskurs erleichtert es, die Verantwortung vom Norden zum Süden zu verschieben. Bevölkerungen, die möglicherweise vom Klimawandel betroffen sein werden, sollen selbst für ihre Sicherheit sorgen. Tatsächlich wird es sich bei der klimawandelbedingten Migration meistens um eine Binnenmigration handeln, innerhalb der Grenzen desselben Nationalstaates “ (Oels, 2017, S. 76).

Implizit erfolgt damit die Abgabe politischer, ökologischer und wirtschaftlicher Verantwortung an Regionen und deren lokale Administrationen, während im Zuge der Globalisierung Konzerne und Finanzdienstleister grenzüberschreitend tätig sind. Katastrophen als solche werden in ihrer Wirkmächtigkeit auch verleugnet, als könnte das resiliente Individuum oder die Gruppe unbeschadet aus solchen Ereignissen hervorgehen. „Sozio-ökologische Resilienz meint nicht nur 
die Überlebensfähigkeit von Gesellschaften, sie zielt zudem auch darauf ab, dass sich diese angesichts dramatischer externer Veränderungen positiv weiterentwickeln" (Oels, 2017, S. 63).

Problematisch ist auch der normative Gebrauch der Begriffe. Vielfach gewinnt man den Eindruck, als wären Resilienz und Anpassung an sich etwas Gutes. In diesem Zusammenhang fließen immer wieder auch Gedanken vom „Survival of the Fittest“" ein:

„Leitfiguren sind nicht die Schwächsten, die Vulnerablen, sondern die Stärksten, die Resilienten: Man schaut auf ihre Ressourcen, Bewältigungsmechanismen, Überlebensstrategien, Unterstützungsnetzwerke, ihr, soziales Kapital' usw., also auf ihre Resilienzfaktoren " (Merk, 2017, S. 136).

Ottomeyer führt in der Bewertung verschiedener Resilienzansätze zum Beispiel ins Treffen, dass Aaron Antonovsky in Bezug auf den von ihm entwickelten „Sense of Coherence“, mit dem Widerstandsressourcen von Menschen gemessen werden können, selber daran zweifelte, ob es möglich ist, zu unterscheiden, ob ein „SOC“ auf Abwehr gegründet oder authentisch ist. Auch der Folterer von Treblinka habe ein neues Leben in Paraguay aufbauen können, und Jugendliche in fundamentalistischen Gruppen würden ein starkes Kohärenzgefühl erleben (vgl. Ottomeyer \& Reddemann, 2017, S. 44). Mit anderen Worten, Resilienz selbst ist kein moralischer Indikator. Im Bereich der Ökologie wird zum Beispiel gefordert, dass das Konzept der Resilienz durch andere Konzepte ergänzt werden muss. „Für emanzipatorische Strategien sind explizite normative Begriffe erforderlich, für die sich Ansätze wie das Konzept der Nachhaltigkeit oder auch der Begriff der Klimagerechtigkeit weitaus besser eignen“" (Hummel, 2017, S. 121).

Dasselbe gilt auch für den Bereich des sozialen Handelns. Auch hier wären Konzepte nötig, die in die Zukunft gerichtete Modelle eines guten Lebens und nicht nur solche des Überlebens beinhalten, während, wie im nächsten Kapitel dargestellt, genau das Gegenteil evoziert wird.

\subsubsection{Resilienz und Sicherheit}

Neocleous (2017) stellt in seiner Auseinandersetzung mit dem Resilienzkonzept dasselbe aus dem Blickwinkel der Kritischen Theorie infrage und erläutert die Auswirkungen eines solchen Denkens auf die Antizipation der Zukunft: „Resilienz ist also nichts anderes als eine Vorwegnahme der Zukunft, doch sie beschwört eine Zukunft, die zunächst als Katastrophe bzw. Angriff ausgemalt wird und dann, was noch wichtiger ist, als eine Erholung von davon" (Neocleous, 2017, S. 157).

Welche Wirkungen ein solches Denken auf das Individuum hat, kann man anhand der Interviewergebnisse gut nachvollziehen, wo Vorstellungen über die Zukunft als beängstigend und belastend erlebt werden, besonders was die Kinder 
und nachfolgenden Generationen betrifft (Kapitel 8.2.9). Diese Bilder generieren Fluchtgedanken und Rückzugsphantasien auf der einen Seite, und Vorstellungen, die um gewaltsame Auseinandersetzungen um Hab und Gut und Ressourcen wie Wasser kreisen, auf der anderen Seite.

Hauptkritikpunkt für Neocleous ist eine Art Kolonisierung des Denkens und der Vorstellungskraft, indem Resilienz uns davon abhält, „über politische Alternativen nachzudenken“ (Neocleous, 2017, S. 152).

„Jede Sorge um den Zustand der Welt und um unseren Platz darin führt unweigerlich zum Resilienztraining, nicht zum politischen Kampf. Man erlaubt uns die individuelle und kollektive Unsicherheit, sowie die persönliche und strukturelle Resilienz. Doch man erlaubt uns nicht, politisch dagegen aktiv zu werden" (Neocleous, 2017, S. 160).

Neocleous beschreibt, in welcher Form das Konzept Eingang in die Diskussion um nationale Sicherheit gefunden hat, und macht darauf aufmerksam, dass das Resilienztraining zum Programm der US-Streitkräfte gehört und nicht-militärische staatliche Organisationsstrukturen und internationale Organisationen und Finanzsysteme darauf zurückgreifen. ${ }^{121}$

Bedrohung und eine „Kultur des Vorbereitet-Seins“ (Neocleous, 2017, S. 156) werden zum Normalzustand. Verborgen werden nach Neocleous die Interessen, die damit zusammenhängen, nämlich dass es letztlich um den „subjektiven Umgang sowohl mit der Ungewissheit und der Instabilität des modernen Kapitals als auch mit der Unsicherheit der nationalen Sicherheit" (Neocleous, 2017, S. 160) gehe.

Abschließend ist zu bemerken, dass die hier behandelten Kritikpunkte an der Instrumentalisierung des Konzeptes der Resilienz nicht darüber hinwegtäuschen sollten, dass in Österreich die Folgen der Finanz- und Wirtschaftskrise gerade wegen bestimmter Maßnahmen und sozialstaatlicher Sicherungssysteme abgefedert werden konnten. So hat sich

,,in Österreich im gesamten Zeitraum seit Eintritt der Krise jene Bevölkerungsgruppe fast kontinuierlich und deutlich verringert [...] die als von Armut oder sozialer Ausgrenzung bedroht definiert wurde. Dieser europaweit seltene Erfolg ist nicht zuletzt auch Ergebnis eines starken sozialstaatlichen Netzes, deren [!] Sozialleistungen Armutsgefährdung aktuell fast halbieren. Darüber hinaus ist zudem kein Anstieg extremer Armutslagen feststellbar. Diese Entwicklung stellt eine wichtige Voraussetzung dafür dar, dass kein krisenbedingtes Erodieren sozialer Sicherheit stattfindet" (Bundesministerium für Arbeit, 2017, S. 376).

121 „Dass das Resilienzkonzept in den höheren Ebenen [der] Staatsmacht auftaucht, spiegelt sich auch darin, dass es zunehmend von internationalen Organisationen wie dem Internationalen Währungsfonds (IWF) und der Weltbank verwendet wird" (Neocleous, 2017, S. 152). 


\subsection{Die künstlerische Verarbeitung der Finanzkrise: ausgewählte Beispiele von Spielfilmen und Dokumentationen}

In diesem Kapitel soll der Blick auf einige Spielfilme und Dokumentationen gelenkt werden, die sich thematisch mit der Finanzkrise auseinandersetzen. Zwei davon stammen aus der ersten Hälfte des 20. Jahrhunderts und beziehen sich auf die Bankenkrise in den 30er-Jahren.

Die Filme stellen für mich eine wertvolle Ergänzung zum Verständnis der Krise dar, weil sie die Suche nach den Ursachen derselben und nach möglichen Auswegen mit den Mitteln dieser Kunstform zu beantworten versuchen. So gesehen verbinden sie sich mit der Konzeption der Interviews, mit denen ich ein ähnliches Ziel verfolgt habe. Filme stehen auf verschiedene Weise dem Unbewussten näher als das der Realität verpflichtete wissenschaftliche Denken. ${ }^{122}$

Das Kino kann als

„Produktionsstätte bewusster und unbewusster gesellschaftlicher Mythen verstanden werden, als ,Apparat' gesellschaftlichen Denkens im Sinne eines Transformations- und Verarbeitungsmediums von sozialen Konflikten, von Geschlechterbeziehungen oder von historischen Traumata, weil es wie keine andere Kunst im öffentlichen Raum rezipiert wird [...] “ (Zwiebel \& Maler-Bungers, 2007, S. 18).

Nicht von ungefähr wird der Kinosaal, abgedunkelt und einer Höhle ähnlich, als Ort empfunden, in dem sich primär- und sekundärprozesshaftes Denken, angeregt durch das Geschehen auf der Leinwand, verbinden. So haben die Zuseher auch die Möglichkeit, das Tabuisierte unmittelbar zu genießen, das Verbotene identifikatorisch zu erleben. Im Krisendiskurs kommen diese Bereiche viel zu kurz. Den Schaden und das Leid vor Augen wird dieser Diskurs nach einer Krise mehr vom Über-Ich beherrscht, das Begrenzung und Kontrolle einmahnt. Die Figur des Traders dagegen stellt eine Verkörperung von Omnipotenz dar; ihm sind scheinbar keine Grenzen gesetzt, er kann unbeschränkt über Geld, Immobilien und Frauen verfügen und so die Gebote der Kultur übertreten, die für die meisten Zuseher gelten.

Freud schreibt dem Phantasieleben einen erheblichen Stellenwert zu, wenn es um die Befriedigung kulturell verpönter Bedürfnisse geht: ,[...] es wurde seinerzeit, als sich die Entwicklung des Realitätssinnes vollzog, ausdrücklich den

122 In den letzten Jahren ist eine Reihe von Büchern erschienen, die sich mit psychoanalytischen Filminterpretationen beschäftigen: Piegler (2008), Wollnik (2008) sowie Zwiebel und Maler-Bungers (2007). 
Ansprüchen der Realitätsprüfung entzogen und blieb für die Erfüllung schwer durchsetzbarer Wünsche bestimmt“" (S. Freud, 1930a).

Handlungen, die mit Entgrenzung, Maßlosigkeit, (Verfügungs-)Macht oder Sexualität und Gewalt assoziiert werden, können im Kinosaal erlebt werden, ohne dass man dafür in der Realität belangt werden könnte. Das ist bedeutsam, weil der Film so auf das individuelle und gesellschaftliche Unbewusste verweist. Er steckt das Terrain ab, innerhalb dessen sich das Verbotene realisieren darf, und öffnet es gleichzeitig auf einer Metaebene für eine gesellschaftskritische Betrachtung und Analyse.

„Als Medium des gesellschaftlichen Unbewussten kann das Kino sowohl affirmativer wie kritischer Kulturpraxis dienen und als Bildmedium im wörtlichen Sinne (positive wie negative) Visionen ${ }^{123}$ gesellschaftlicher Zustände im Sinne einer selbstreflexiven kulturellen Sinngebungspraxis entwerfen" (Zwiebel \& MalerBungers, 2007, S. 19).

Ich werde stellenweise die Darstellung der Filminhalte durch entsprechende Analysen, den Bereich der Finanzwirtschaft betreffend, ergänzen.

\subsubsection{Spielfilme}

Der Film „American Madness“ (Capra, Dwan \& Neill, 1932, USA) veranschaulicht in ausdrucksstarken, einprägsamen Bildern, was bei einem Bankensturm geschieht. Im Zentrum dieses Filmes steht der Vertrauensverlust in die Liquidität einer Bank. Gleichzeitig werden jedoch auch unterschiedliche wirtschaftspolitische Konzepte verhandelt, die gegenwärtig eine große Rolle spielen: Sollte volkswirtschaftlich eine ausgabenorientierte oder eine sparsame Politik verfolgt werden? Diese Kontroverse bildet den Hintergrund, vor dem sich das Geschehen ereignet.

Der Bankier Tom Dickson verfolgt - sehr zum Unmut des Vorstandes, der ihn zum Rücktritt bewegen will - eine Kreditvergabepolitik, die das Geld in Umlauf bringt und die Wirtschaft florieren lässt. In einer fulminanten Rede vor dem Vorstand erklärt er seine Grundsätze: „Ich bin nicht am Profit, ich bin an der Bank interessiert". Als sich, ausgelöst durch einen Banküberfall, rasend schnell Gerüchte über fehlende Reserven der Bank verbreiten, kommt es zu einem Massenansturm der Kunden, zu Tumult und Panik. Das neue Medium Telefon wird zum Multiplikator der Ängste, die sich in den Naheinstellungen der Gesichter neben einer Mischung aus Erregung, Panik und Lust widerspiegeln. Das Telefon ist ein Symbol für den technischen Fortschritt und für eine zum damaligen Zeit-

123 Hervorhebung durch die Autoren. 
punkt noch unbekannte Geschwindigkeit in der Nachrichtenübermittlung, die mit der heutigen Computertechnologie vielfach gesteigert wurde. Ein Meer wogender Hüte, aus der Vogelperspektive gefilmt, brandet an die Mauern des Bankgebäudes und überflutet die Innenräume. Verzerrte Gesichter und eine gewaltsame Sprache verdeutlichen den Verlust an Rationalität. Die Bilder einer hysterisierten Masse sind jene, die noch nach Jahrzehnten mit dem Börsenkrach assoziiert werden und transgenerational, wie man auch in einem Interview (siehe Seite 227) sehen kann, auftauchen.

Zwei Filme aus der jüngsten Vergangenheit beschreiben auf unterschiedliche Weise die Zeit kurz vor der Finanzkrise. Gewinner und Verlierer begegnen einander. Der erste, „The Big Short“ (McKay, 2015, USA), erzählt, wie mehrere Männer unterschiedlichen Alters und unterschiedlicher Einstellungen, allesamt im Bereich des Finanzsektors als „Trader“ tätig, die Immobilienkrise im Jahr 2008 vorhersehen und so das große Geld machen. „Sind Sie Drogendealer oder Banker?", diese Frage eines Durchschnittsbürgers in einem englischen Pub an einen ehemaligen Bankmanager, bleibt von diesem unbeantwortet. Sie könnte als Motto des Films gelten.

Während der Bankensektor in den 70er-Jahren, so die Erzählerstimme von Jared Vennett am Beginn des Films, ein langweiliges Geschäft war, wo man mit Anleihen ein paar Dollars verdienen konnte, sollte sich das durch die neuen Finanzprodukte gravierend ändern.

Dr. Michael Burry, ein halbblindes Genie, sieht die Immobilienblase voraus und kauft Ausfallshaftungen bei den großen Banken wie der Deutschen Bank oder Goldman Sachs. Er will damit die Anleihenpakete „shorten“, das heißt, gegen sie wetten. Mark Baum, der seinen Bruder durch Suizid verloren hat, wird von Schuldgefühlen geplagt, weil er dem Bruder Geld statt Hilfe angeboten hat. Er ist der Ankläger von Ungerechtigkeiten und Widersprüchen aller Art, so, als könnte er dadurch seinen eigenen Versäumnissen entkommen. Er verwaltet mit drei Mitarbeitern einen Fonds bei Morgan Stanley und wird durch Zufall darauf aufmerksam, dass CDOs (Collaterized Debt Obligations) Pakete von hochriskanten Darlehen sind.

Jared Vennett, ein unsympathischer, egozentrischer Mann, der bei der Deutschen Bank beschäftigt ist, dort wenig Ansehen genießt und Geld verdienen möchte, bringt Mark Baum und seine Mitarbeiter dazu, Geld in Credit Default Swaps zu investieren. Vennett lässt sich durch nichts aus der Ruhe bringen, er strahlt ein überbordendes Selbstbewusstsein aus, das durch die sadistische Behandlung seines Assistenten ständig Nahrung bezieht.

Zwei junge Männer, Charlie Geller und Jamie Shipley, Gründer des Brownfield Fonds, möchten mithilfe des befreundeten, ausgestiegenen Bankers bei Morgan Chase, Ben Rickert, reich werden. Sie setzen die Idee um, die ,gerateten“ Produkte zu ,shorten“, was ihnen ein Vermögen einbringt. 
Die Dialoge der unter Druck stehenden Protagonisten erzeugen im Zuseher ein Gefühl von Druck, Abwehr und Aggressivität: „Leck mich am Arsch!“, „Sie sind ein Haufen Scheiße!“, „Das ist ein Scheißbetrug!“, „Zeit, dass die Scheiße auffliegt!“”, „Sie sagen mir, wie viel ich wert bin, und Sie sagen mir, wie viel Sie wert sind!“'124 Überhaupt ist die Finanzwelt, so scheint es, eine Männerwelt, die sich zum Ausgleich für ihre Tätigkeit heftiger Verbalinjurien, eines intensiven Fitnesstrainings oder der Prostitution bedient. ${ }^{125}$

Die treibende Kraft des Gewinns ist, so einer der Protagonisten, die Dummheit oder, besser gesagt, die Verleugnung, weil selbst die Kenner des Systems ihr Vertrauen in ihre Umgebung nicht verlieren wollen. Sie schreien sich gegenseitig an oder jammern „Das ergibt keinen Sinn!“, weil sie den umfassenden Betrug nicht erkennen wollen. ${ }^{126}$ Es geht neben dem Betrug an anderen immer auch um Selbstbetrug. Dazu kommt die Heuchelei, wie eine Repräsentantin von Standard \& Poor's, einer führenden Ratingagentur, Mark Baum zu verstehen gibt, der sie des Betrugs anklagt, weil sie für wertlose Derivate Triple-A-Ratings vergeben haben, um keine Kunden an die Konkurrenz zu verlieren. Baum selbst, so die Raterin, verdiene aber das große Geld mit CDS (Credit Default Swaps). Damit thematisiert der Film auch Spaltungsprozesse, die durch die unterschiedlichen Charaktere verkörpert werden. Gier, Dummheit, Heuchelei und Verleugnung führen sowohl bei Gewinnern und als auch Verlierern dazu, dass, so das Resümee des Films, sich das Karussell weiterdreht, unabhängig davon, wer auf den Pferden sitzt bzw. wer schon den Platz geräumt hat. Im Vergleich zu „American Madness" ist nur die Geschwindigkeit viel größer geworden.

Der zweite Film, „Margin Call“ (Chandor, 2011, USA), spielt kurz vor dem Ausbruch der Krise und thematisiert, wie in der Finanzszene mit Verlusten umgegangen wird.

Knapp vor dem Beginn der großen Bankenkrise 2007/2008 entdeckt der Risikomanager einer New Yorker Bank, Eric Dale, dass in den nächsten Tagen der Zusammenbruch derselben bevorsteht, da das Risikopotenzial von Wertpapieren, von denen die Bank viel zu viele besitzt, falsch bewertet worden ist. Er wird trotz großer Kompetenzen nach 19 Jahren Beschäftigung entlassen, kann aber zuvor noch seinem Mitarbeiter Peter Sullivan einen USB-Stick mit den brisanten Daten übergeben. Kündigungen stehen schon länger auf dem Plan.

124 Sie stellen einen offensichtlichen Bezug zwischen Analität und Geld her.

125 Das ist, wie mir von ehemaligen Tradern versichert wurde, durchaus realitätsnah dargestellt.

126 Tuckett hat dieses Verhalten als typisch für Vermögensverwalter beschrieben, die mit sogenannten volatilen Produkten handeln. 
Fehlende Solidarität und Unberechenbarkeit machen den Arbeitsplatz selbst zu einem volatilen Feld.

Der drohende Arbeitsplatzverlust wird durch zwei Frauen verkörpert, die mit einer Delegation von Helfern durch die Großraumbüros schreiten und die Kündigungen vollziehen. Es bleibt unklar, ob sie Teil des Firmenimperiums sind oder einer externen Personalberatungsfirma angehören. Verhandelt wird nicht. Niemand kann vorhersagen, wer an die Reihe kommt. Ein führender Angestellter, Will Emerson, sagt zu seinen Kollegen: „Schaut einfach nicht hin.“ Verleugnung und mangelndes Wissen sind in diesem Film zentral. Die Erleichterung, selbst nicht zu denen zu gehören, die binnen einer halben Stunde das Gebäude mit einem Karton verlassen müssen, paart sich mit dem im anschließend einberufenen Meeting erfolgenden Aufruf, sich gegen die Gegner - das sind die Kollegen - durchzusetzen.

Der Zuseher wird Zeuge eines von vielen Ritualen: Das Meeting wird vom Vorgesetzten Sam Rogers und seinen Mitarbeiten durch Applaus eingeleitet, so als gäbe es etwas zu feiern. Der Zuseher fragt sich, ob dies ein Überbleibsel aus jenen Zeiten ist, als jeder von den Angestellten Gewinne machte, oder ein Beklatschen des Faktums, dass man nicht zu den Gekündigten gehört. Möglicherweise ist es auch eine agierende Abwehr. ${ }^{127}$

Weitere Rituale sind schnelle Fahrten mit teuren Autos, gemeinsamer Alkoholkonsum in schicken Bars und der Kontakt mit Prostituierten, um die Spannungen abzubauen. Ungläubig staunen die Neuangestellten über die astronomischen Summen, die Vorgesetzte verdienen. Der CEO der Bank, John Tuld, sagt lakonisch: „Was ist schon Geld. Papier mit aufgedruckten Köpfen.“ Geld hat keinen eigentlichen Wert.

Doch selbst im Niedergang gibt es bedeutende Unterschiede. Wer den Kündigungen widerspruchslos zustimmt, kann mit kurzfristiger Fortzahlung der Krankenkassenbeiträge und einer guten Abfindung rechnen. Wer über Wissen verfügt und schweigt, wie der gekündigte Analyst, wird mit horrenden Summen belohnt.

Die Verkaufspraktiken sind skrupellos und entbehren jeder Verantwortung. Die wertlosen Papiere werden an Bekannte und Familienmitglieder verkauft, um die Boni zu beziehen. Die scheinbare Loyalität gegenüber der Firma, die dieses Verhalten mit hohen Summen belohnt, ist stärker als die Verbundenheit mit Freunden und Familienmitgliedern. Auch im Niedergang gilt es, so viel Geld

127 Reflexion findet jedenfalls nicht statt. In Bezug auf die von ihm interviewten Vermögensverwalter konstatiert Tuckett Verleugnung und Vorwurf als bevorzugte Abwehrmechanismen und schreibt: „Sie [Die Vermögensverwalter] beklagten sozusagen ihr Scheitern nicht, sondern waren vielmehr bemüht, weiterzumachen und sich für den nächsten Kampf zu rüsten“" (Tuckett, 2013, S. 258). 
herauszuholen wie möglich. Es wird in Kauf genommen, dass dies mit einem absoluten Vertrauensverlust der Kunden in die Bank verbunden sein wird. Die Verantwortlichen wissen jedoch, dass sie dann genug verdient haben werden. In dieser Welt hat mathematisches Wissen nur insofern einen Stellenwert, als es aufzeigt, wie unwahrscheinlich es ist, dass man auf Dauer gewinnen kann. Einer der Angestellten bringt es auf den Punkt: Es ist Glücksspiel, das die Mitarbeiter betreiben, mit dem Unterschied, dass die Vorgesetzten das wissen, die hoffnungsfrohen Neuankömmlinge jedoch nicht.

So wie diese Männer über die Dächer von Wolkenkratzern schauen, wo der Blick in die Tiefe der Häuserschluchten den möglichen eigenen Sturz in den Abgrund vorhersieht, verkörpern sie auch das Abgehobensein vom „normalen“ Leben, das sie jedoch durch ihre Entscheidungen wesentlich mitbestimmen. Auch „normale“ Leute sind Spieler, wenn sie den Versprechen Glauben schenken, schnelles Geld verdienen zu können, ohne zu fragen, auf welchen Grundlagen das passiert.

Eine Botschaft des Films ist, den Blick historisch zu erweitern. Der Eigentümer zählt lakonisch, im frühen 19. Jahrhundert beginnend, die Krisenjahre auf, und macht sie damit zu einem regelmäßigen Bestandteil des wirtschaftlichen und sozialen Lebens im Kapitalismus. Im Sinne dieses Wirtschaftssystems handeln auch die Protagonisten: Wenn sie die Möglichkeit haben, viel Geld zu verdienen, entscheiden sich selbst diejenigen, die moralische Bedenken haben, gegen die Moral. Eine weitere wichtige Botschaft des Films ist, dass skrupelloses und systemkonformes Verhalten belohnt wird. ${ }^{128}$

Die Frauen im Film sind einerseits die ausführenden Organe für unangenehme Entscheidungen wie Kündigungen, sie verkörpern aber auch die gekaufte Lust. Meistens sind sie die Verlierer in diesem Geschäft. Sie werden geopfert, wenn sie einen männlichen Konkurrenten haben, oder bleiben in Beziehungen auf der Strecke, wie im Fall der Ehefrau von Sam Rogers. Es bleibt dem Zuseher überlassen, Letzteres als Vor- oder Nachteil anzusehen.

Im Film „The Company Men“ (Wells, 2010, USA) verfolgt man die Verlierer der Krise bei ihrem Absturz. Dabei thematisiert der Film klassenspezifische Ge-

128 Michael E. Shulman thematisiert die weitläufige Verdrängung der Krise in den USA, wie sie auch in der Rezeption des Films zum Ausdruck kommt, in einer Fußnote. "The remarkable lack of commercial success in the United States of a superbly-cast, -scripted and -reviewed film based on the beginning of the crisis, Margin Call, also bears witness, in a different way, to American anxieties in contemplating the financial crisis. The film, starring some of the best-known actors in Hollywood, grossed only $\$ 5.4$ million in the United States, and more than twice that overseas" (Siehe dazu die Fußnote in Shulman, 2016, S. 48). 
gensätze und greift auch die Frage nach einer Lösung der Krise auf, die die Sehnsucht nach einer verlorenen Zeit widerspiegelt.

Im Zentrum steht die Verlusterfahrung des Mittelstands, zunächst festgemacht an materiellen Verlusten wie Autos oder Häusern. Dazu kommt der Verlust der Kreditwürdigkeit und des gewohnten Lebensstils. Die beschäftigungslosen Männer schämen sich und versuchen, ihre Arbeitslosigkeit vor ihrer Familie und den Nachbarn zu verbergen. Sie treffen einander in „Outsourcing Companies“, wo sie sich in speziellen Trainings Selbstbewusstsein und eine erfolgreiche Zukunft suggerieren sollen. Einer von ihnen, Phil Woodward, ist sechzig und hat kaum mehr Chancen auf einen Arbeitsplatz, auch wenn er sich das Haar färbt. Die Verluste führen zu depressiven Zuständen und gehen mit Aggressionen einher. Alkoholkonsum und Aggressionsausbrüche in Form von Beschimpfungen der weiblichen Vorgesetzten, die die Kündigungen vollzieht, oder einer Frau, die zum Bewerbungsgespräch lädt, sind auffallend stark sexuell konnotiert oder körperlich entwertend: Die Frauen werden als Schlampe bezeichnet, oder es wird auf ihr Übergewicht angespielt.

Phil Woodward, der sich das Leben nehmen wird, wirft in der Nacht aus Verzeiflung und Wut über seine ausweglose Situation Steine auf das ehemalige Firmengebäude, das er wegen der Abschottung durch Zäune nie treffen wird. Er verkörpert ein Bild der Hilflosigkeit.

Der Film beschreibt die psychischen und physischen Folgen der Beschäftigungslosigkeit und ihrer Auswirkungen auf das soziale Umfeld. Zwischen den „Company men“, zu denen der Hauptdarsteller Bobby Walker gehört, und der Klasse der Handwerker scheinen unüberbrückbare Gegensätze zu herrschen: Voller Verachtung blickt man auf den jeweils anderen. Klassengegensätze scheinen unverrückbar. Damit thematisiert der Film Abgrenzungsversuche, die der Identitätsdiffusion infolge der mannigfaltigen Verlusterfahrungen entgegenwirken sollen. Das Handwerk, verkörpert durch Bobby Walkers Schwager Jack Dolan, scheint in Krisenzeiten noch zu florieren, obwohl Dolan viel weniger verdient.

Walker selbst hofft, in einem neu gegründeten Unternehmen Arbeit zu finden. Diese Hoffnung verweist auf die Schwerindustrie, wo ein Neuanfang gestartet werden soll, ist jedoch nicht nachvollziehbar: Eine alte Schiffswerft in verrottetem Zustand soll wieder in Betrieb gehen. Dabei ist für den Zuseher offensichtlich, dass diese Produktionsstätten längst ins Ausland ausgelagert wurden.

Hier ist auch nach der Krise keine Veränderung in Sicht. Das Rad dreht sich weiter, ohne dass sich das Bewusstsein verändert hätte. Die Menschen im Film versuchen, sich in der Vergangenheit zu verankern, um nicht gänzlich den Boden unter den Füßen zu verlieren. Weder Handwerk noch Industrie können in jenen Zeiten als Hoffnungsträger gelten, in denen die wirtschaftlichen Strukturen durch die Globalisierung tiefgreifend verändert wurden. 
Der nächste Film, der zu den Dokumentationen überleitet, welche das Geschehen, das zur Finanz- und Staatsschuldenkrise in Europa geführt hat, aufrollen, stammt aus dem Jahr 1940. Er erzählt auf subversive Art und Weise über die Verflochtenheit von Politik und Kapital und den moralischen Verfall einzelner Funktionsträger in diesen Bereichen.

Im Zentrum von „The Great McGinty“ (Sturges, 1940, USA) steht der krisenhafte Moment im Leben zweier Männer. Es sind dies Dan McGinty, der mithilfe der mafiaähnlichen Organisation des Politikers Skeeters vom Wahlbetrüger, Geldeintreiber und Bürgermeister bis hin zum Gouverneur einen kometenhaften Aufstieg zu verzeichnen hat; der andere ist der Bankkassier Thompson, der sich aufgrund eines Betrugs das Leben nehmen möchte. Sie treffen einander in einer Bar, in der McGinty, fern von seinem früheren Leben, mit seinen alten Weggefährten als Barkeeper arbeitet und den unglücklichen Banker mit der Erzählung seiner eigenen Lebensgeschichte vom Selbstmord abhalten kann. In einem Rückblick erfährt man Folgendes:

In dem Moment, in dem McGinty, beeinflusst von seiner Frau Catherine, sich entschließt, aus dem korrupten Politikergeschäft auszusteigen, wendet sich das Blatt. Er wird beinahe Opfer eines Anschlags, kommt dann wegen der aufgedeckten früheren Machenschaften ins Gefängnis, kann aber letztlich mithilfe des Politikers, dem er durch Wahlbetrug zum Sieg verholfen hat, das heißt gemeinsam mit dem ehemaligen Mafiaboss fliehen. Seine Ehefrau Catherine, die als Einzige die Moral verkörpert, bekommt von ihm noch einen telefonischen Hinweis, wo sich das versteckte Geld befindet.

Die entlastende Botschaft für den Bankkassier lautet: Ehrlichkeit führt zum Fall. Daher solle er sich wegen eines Betrugs nicht das Leben nehmen. Selbst die „Guten“ wie Catherine sind Nutznießer von korrupten Geschäften. So gesehen hat der Film eine subversive Botschaft: Moral und wirtschaftlicher, aber auch politischer Erfolg sind unvereinbare Gegensätze.

\subsubsection{Dokumentationen}

Dokumentationen sind per se mehr dem Realitätsprinzip verpflichtet, da sie mit Daten und Fakten operieren. Nichtsdestotrotz spiegeln die Auswahl derselben, ihre Zusammenstellung und gestalterische Elemente wie Musik den Blickwinkel des Regisseurs wider und beeinflussen so den Zuseher. Natürlich handelt es sich bei den folgenden Beispielen auch um eine durch die Zielsetzung dieser Arbeit bedingte Auswahl. ${ }^{129}$

129 Die Dokumentationen von Ferguson (2009), Roche und Fritel (2013) sowie Stucke (2009) habe ich nicht in die Besprechung aufgenommen, da sie mir wegen ihres einführenden oder reißerischen Charakters nicht adäquat erschienen. 
Nach der Finanzkrise entstand eine Fülle an Dokumentationen, die, dem Wunsch nach Aufklärung im historischen Sinn geschuldet, eine Gegenposition zur Lähmung der Öffentlichkeit einnahmen. Aber nicht nur das: Sie zeigten auf, dass das Unverständliche und Abgehobene, das mit dem Derivatehandel und der Globalisierung des Finanzsektors oft assoziiert wird, der Verschleierung von unlauteren Geschäftspraktiken dient. Damit tragen diese Filme dazu bei, dass das soziale Unbewusste zum Teil bewusst gemacht wird.

Im Spannungsfeld zwischen Spielfilmen und Dokumentationen fällt den Dokumentationen - bezieht man sich auf die psychischen Instanzen - mehr die Position des Ichs bzw. vor allem des Über-Ichs zu. Das Verbotene, das man in den Spielfilmen genießen kann, wird in den Dokumentationen in seinen realen Auswirkungen stärker ins Zentrum gerückt. Das Über-Ich versetzt nach Freud das Ich in Angst und Schrecken vor Strafe, unabhängig davon, ob die verbotene Tat ausgeführt oder nur vorgestellt wird:

„Es wird also trotz des erfolgten Verzichts ein Schuldgefühl zustande kommen, und dies ist ein großer ökonomischer Nachteil der Über-Ich-Einsetzung, wie man sagen kann, der Gewissensbildung “ (S. Freud, 1930a, S. 254).

Wie schon im Kapitel über die metapsychologischen Konzepte des Unbewussten ausgeführt wurde, ist das Über-Ich tief im Es verwurzelt und reagiert infolge der Triebentmischung oft mit großer Unerbittlichkeit und Härte. Verfolgt man die Dokumentationen, werden unweigerlich innerpsychische Prozesse in Gang gesetzt, die neben dem Erkenntnisgewinn sehr oft vom Über-Ich geleitete Bestrafungswünsche auslösen.

Ein Paradebeispiel dafür sind die Filme von Michael Moore, der mit seinem Film „Capitalism: A Love Story“ (Moore, 2009, USA) eine kapitalismuskritische Rezeption intendiert, durch seine einseitige Darstellung aber oft das Gegenteil bewirkt. Nichtsdestotrotz gelingen ihm aufklärende Momente, etwa wenn er Wirtschaftsprofessoren in einen Erklärungsnotstand bringt, indem er sie bittet, den Begriff und die Funktion von Derivaten zu erklären, womit er auf das Numinose dieser Finanzprodukte verweist.

Auch Politikern auf höchster, mittlerer und unterster Ebene fehlt das finanztechnische Wissen, sie werden jedoch durch persönliche finanzielle Vorteile zum Beispiel durch Kredite ohne Auflagen oder Geldflüsse zu Gemeinden für privat geführte Haftanstalten für Jugendliche - zu Nutznießern des Systems. Es ist auch kein Verlass auf die Rechtsprechung, so eine der Botschaften des Films, wie am Beispiel zu Unrecht verurteilter oder zu lange in Haft bleibender Jugendlicher aufgezeigt wird. Die Richter sind ebenfalls durch Gewinnstreben korrumpiert. Immobilienmakler, „Aasgeier“ im Film, bereichern sich an verschuldeten Menschen, und Politiker wie G. W. Bush nützen 9/11, um von der verheerenden Wirtschaftskrise abzulenken und alte Bündnisse mit Bankern, die zu einer mas- 
siven Umverteilung von unten nach oben führten, wieder zu festigen. Ein weiteres Beispiel für die Entgleisungen des Kapitalismus sind jene Konzerne, die Lebensversicherungen auf Mitarbeiter abgeschlossen haben, ohne dass diese etwas davon wussten. Die Familien der sogenannten „dead peasants“ gingen leer aus. ${ }^{130}$

Der Kapitalismus wird in der Dokumentation jedoch wiederholt personifiziert, damit mystifiziert und erhöht. Das konterkariert die realen negativen Auswirkungen des Wirtschaftssystems, die Moore aufzeigt und die reale Handlungsmöglichkeiten zuließen. Bedingt durch die fast holzschnittartige Zeichnung des Films werden auch Spaltungsprozesse bedient, die zwischen „Tätern“ und „Opfern" changieren und zum Beispiel die individuelle Verleugnung der fehlenden Kreditwürdigkeit der Betrogenen nicht zum Thema werden lassen.

Im Dokumentarfilm „The Forecaster“ (Vetter \& Steinberger, 2015, USA) wird die Geschichte von Martin Armstrong erzählt, der bereits von Kindheit an eine Affinität zu Münzen und Computern entwickelt und vom Münzhändler zum Leiter von „Princeton Economics“ aufsteigt, einer Firma, die aufgrund ihrer Voraussagequalitäten in Bezug auf Finanzkrisen und kriegerische Konflikte zu einem gefragten Berater von Banken und Händlern wird. Vom Jahr 1999 an wird für Armstrong ohne Anklage sieben Jahre lang die Beugehaft verlängert, um ihn zur Herausgabe von Münzen, einer Cäsarbüste und eines Computers, auf dem sich die Geheimformel zur Berechnung der Wirtschaftskrisen befinden soll, zu bewegen. Vor die Wahl gestellt, ein Geständnis zu unterschreiben, an einem großen Finanzbetrug beteiligt gewesen zu sein, oder das ganze Leben im Gefängnis zu verbringen, entschließt sich Armstrong für Ersteres. Daraufhin wird die Haft ohne Beweise und Zeugeneinvernahmen um weitere fünf Jahre verlängert.

Zwei zentrale Themen kristallisieren sich im Laufe der Dokumention heraus: einerseits die Missachtung von Recht und andererseits die Frage nach der Möglichkeit, durch das genaue Studium von wirtschaftlichen Entwicklungen über lange Zeitperioden hinweg mehr Wissen über die Gesetze des Marktes zu erlangen. Bei Armstrong spielt dabei die Zahl „Pi“ eine große Rolle. Was er jedoch wirklich beschreibt, ist das reale Zusammenspiel von politischen und wirt-

130 Obwohl es so scheint, sind dies nicht Beispiele für einen Werteverlust in unserer Gesellschaft, sondern, wie Verhaege schreibt, es sind gerade die Werte des Kapitalismus, die etabliert werden. "There are constant laments about the so-called loss of norms and values in our culture. Yet our norms and values make up an integral and essential part of our identity. So they cannot be lost, only changed. And that is precisely what has happened: a changed economy reflects changed ethics and brings about changed identity. The current economic system is bringing out the worst in us" (Verhaeghe, 29.11.2014). 
schaftlichen Interessen, die fehlende Regulation des Finanzsektors und damit einhergehende kriminelle Aktivitäten.

Für Freud steht das Unheimliche in Beziehung zur Wiederkehr des Verdrängten (vgl. S. Freud, 1919h, S. 268 ff.).

Worin besteht das Unheimliche in diesem Film? Es ist nicht so sehr das Verschwörungsszenario, das Filmkritiker hier als Baustein eines guten Thrillers ${ }^{131}$ in den Fokus stellen, das in vereinfachender Weise Täter und Opfer der Krisen auflistet und Parteinahme von den Zusehern fordert. Viele Aspekte, die den Finanzmarkt betreffen, erscheinen undurchsichtig, unverständlich und in ihren sozialpolitischen Auswirkungen bedrohlich. Verdrängt und damit unbewusst bleiben muss, dass der Markt kein Organismus ist, der unabhängig von menschlichen Entscheidungen lebt und handelt, sondern dass es reale Entscheidungsträger gibt; unbewusst bleiben muss, dass Rechtssysteme, wenn sie nicht kontrolliert werden, selbst in Demokratien zur Bedrohung für die Freiheit von Individuen und Gruppen werden können; unbewusst bleiben muss weiter, dass es reale Anzeichen dafür gibt, wenn politische Systeme zusammenbrechen, dass zum Beispiel kurz davor vermehrte Geldflüsse ins Ausland zu beobachten sind. Unbewusst muss auch bleiben, dass Institutionen, die zum Schutz von Staaten gegründet wurden, wie zum Beispiel die Weltbank, auch von ihren Akteuren abhängig sind, die interessengeleitet ihren Eigennutz verfolgen. Der Zuseher wird mit Informationen konfrontiert, die er im Sinne einer Verschwörungstheorie lieber abwehren möchte, um die eigene Beteiligung auch in Form des Nichtwahrnehmens nicht reflektieren zu müssen.

In Verschwörungstheorien verdichten sich verschiedene Abwehrmechanismen. Neben der Verleugnung kommt es somit auch zu einer Verschiebung und Spaltung. Diese Abwehr aufzulockern, gelingt nur, wenn man mehr wissen will. Was der Film beschreibt, kann man auch bei Stiglitz (2004) nachlesen, der ohne eine „Welt-“" oder „Geheimformel“" auskommt.

Die Dokumentation „Inside Job“ (C. Ferguson, 2010, USA), die 2011 den Oscar für den besten Dokumentarfilm erhielt, beschreibt in fünf Teilen anhand von Interviews, wie es zwischen 2001 und 2007 zur Immobilienblase und zur Finanzkrise kam, welche Auswirkungen diese Krisen zeitigten und was der Status quo im Jahr 2010 war. Dieser Film lässt viele der einflussreichen Politiker und Wirtschaftsgrößen zu Wort kommen und vermittelt ein faktenbasiertes Wissen.

Als wesentliche Voraussetzungen der großen Finanzkrise, die 2007 in den USA ihren Anfang nahm, werden Deregulierungsprozesse im Finanzsektor ge-

$131 \mathrm{http}: / / w w w . z e i t . d e / k u l t u r /$ film/2015-04/forecaster-dokumentarfilm-armstrong, 05.08.2016; http:/www.welt.de/kultur/kino/article140669298/Das-ABC-der-Ver schwoerungstheorie.html, 05.08.2016. 
nannt. Am Beispiel Islands, das im Jahr 2008 bei einem Bruttoinlandsprodukt von 13 Milliarden Dollar zu Zeiten des Bankrotts der drei privatisierten Banken einen Verlust von 100 Milliarden Dollar zu verzeichnen hatte, werden die Dimensionen der Krise am Beginn des Films sichtbar gemacht.

In den USA setzte nach 40 Jahren wirtschaftlichen Wachstums mit einer streng überwachten Finanzpolitik unter der Ära Reagan die Deregulierung ein. Es kam zu Bankenfusionen, die, sollten die Großbanken in Schwierigkeiten geraten, die ganze Wirtschaft bedrohen konnten. George Soros veranschaulicht den Prozess der Fusionen mit dem Bild eines Öltankers, in dem das Öl nicht wie üblich in voneinander abgetrennten Kammern gelagert ist, sondern sich im Rumpf frei bewegt, sodass das Schiff außer Kontrolle gerät und nicht mehr navigiert werden kann. Die Trennung von Investment- und Geschäftsbanken wurde aufgehoben und die Deregulierung hatte zur Folge, dass Kontrollen reduziert oder gänzlich beseitigt wurden, sodass unlimitierter Spekulation und Betrug Tür und Tor geöffnet wurden. Dies änderte sich weder unter der Regierung Bush noch unter der Regierung Obama, der vor seiner Wahl Versprechen zur Kontrolle der Wall Street abgegeben hatte, aber dieselben Männer in die führenden Stellen der staatlichen Kontrollorgane einsetzte, die schon unter den vorherigen Präsidenten dort federführend gewesen waren.

Die Immobilienblase basierte darauf, dass Kredite an Menschen vergeben wurden, von denen man wusste, dass sie sie nie würden zurückzahlen können; diese Menschen wurden mit dem Versprechen eines Eigenheims gelockt, sich in die Schuldenfalle zu begeben. Die Hypotheken wurden an Investmentbanken verkauft, die die sogenannten Subprime-Kredite sogar bevorzugten, da sie höhere Zinssätze und Prämien abwarfen. Dann wurden neue Finanzprodukte, Derivate, geschaffen, die aus einer Bündelung von Schuldverschreibungen unterschiedlichen Risikos bestanden. Diese wurden an Investoren weiterverkauft. Rating-Agenturen wurden dafür bezahlt, diesen teils hochriskanten Produkten gute Bewertungen auszustellen. Agenturen wie Moody's machten ein großes Geschäft damit, wertlose Produkte wie sichere Staatsanleihen mit Triple A oder AA zu bewerten. Ende der 1990er-Jahre stellten die Derivate einen unregulierten Markt von 50 Milliarden Dollar dar. Je riskanter diese Produkte waren, desto mehr Prämien warfen sie ab.

Die Unverfrorenheit der Banken wurde aber noch größer, als sie begannen, hochriskante Produkte zum Beispiel an Rentenfonds zu verkaufen und gleichzeitig mit CDOs (Collateralized Debt Obligations) darauf zu wetten, dass diese Produkte wertlos würden. Ein anderes Produkt, die CDS, Credit-Default-Swaps, sind Kreditausfallsversicherungen, die auf Produkte abgeschlossen werden, bei denen man nicht selber Kreditgeber ist. Man spekulierte also auf den Ausfall eines Kreditnehmers. All diese Produkte funktionierten nach dem Modell „private gain - public loss“. 
Die immer stärkere Verzahnung von Politik, Bankensektor und Ökonomen an Eliteuniversitäten, ersichtlich am Beispiel von Goldman-Sachs-Vorständen und Führungskräften, wie Alan Greenspan, Martin Feldstein, Larry Summers, Henry Paulson oder Ben Bernanke, Frederic Mishkin im Finanzministerium oder als Leiter der FED bzw. als Notenbankchefs, führte letztlich dazu, dass es zu immensen Umverteilungen innerhalb der Gesellschaft, zu Betrug, fehlender strafrechtlicher Verfolgung der Verantwortlichen und Übertragung der Verluste auf die Bevölkerung kam.

Der Einflussbereich der Banken und Versicherungen erstreckt sich auch auf die Universitäten, wo zukünftige Generationen von Ökonomen ausgebildet werden. Einzelne Professoren beziehen immens hohe Beratergehälter und sehen dabei keinen Interessenkonflikt. 3000 Lobbyisten werden vom Finanzsektor bezahlt, sodass auf einen Kongressabgeordneten mehr als fünf Lobbyisten kommen. Viele Mathematiker und Ingenieure wechselten nach dem Kalten Krieg in den Finanzsektor, weil sie dort Unmengen verdienen konnten.

Was unbewusst bleiben muss oder abgespalten wird, ist, dass im Zuge dieser Jahrzehnte eine Umverteilung des Vermögens zu jenem einen Prozent der Wohlhabendsten der Bevölkerung stattgefunden hat, weiter, dass der Staat, das heißt die Bevölkerung, für die großen Verluste aufkommen muss, die verspekuliert wurden, während die Verantwortlichen bzw. die Gutachter der Ratingagenturen auch nach dem Bekanntwerden des Bankrotts Abfindungen in Millionenhöhe bekamen oder monatlich zweistellige Millionenbeträge als Berater kassierten. Unbewusst bleiben müssen die kriminellen Aktivitäten derer, denen man eigentlich Vertrauen schenkt.

Während Millionen in Amerika und - durch die globale wirtschaftliche Verflechtung bedingt - auch in Europa und Asien ihre Arbeit und ihr Heim verloren, leisteten die Verantwortlichen keine Kompensation. Rechtfertigungen der Verantwortlichen, soweit sie überhaupt Stellung bezogen, waren: „Jeder tut es!“, „Niemand sollte sich auf Analysten verlassen!“”, „Ratingagenturen geben nur ihre eigene Meinung kund!“ Das Bild von einem „Pisswettbewerb“, das im Film als Metapher für die Gier gebracht wird, wenn es um die unvorstellbar großen Summen geht, die verdient wurden, evoziert die Frage: Wer kommt weiter? Drogen, Privatjets, Autos, Luxusvillen und Prostituierte erscheinen wie Fetische, die für ihre Besitzer tot, leblos und unproduktiv sind. So viel davon sie in ihrem Begehren auch anhäufen, so wenig können sie auf Dauer etwas damit anfangen. Die dargestellten Akteure der Finanzszene erscheinen wie perverse Persönlichkeiten, die ihre eigene Kastration ständig verleugnen müssen. ${ }^{132}$

132 Oliver Decker analysiert: „[...] der sexuelle Fetischismus [gibt] im Moment des Scheiterns seines Bemühens Auskunft über etwas Grundsätzliches, was mit der warenproduzierenden Gesellschaft aufs Engste verbunden ist. Das Streben, den mensch- 
Der Titel der Dokumentation legt nahe, dass die Straftaten von Insidern, also von Menschen begangen werden, die Teil des Systems sind und dieses schädigen, indem sie ihr Wissen zum eigenen Vorteil nützen.

Der letzte hier besprochene Dokumentarfilm „Wer rettet wen? Die Krise als Geschäftsmodell auf Kosten von Demokratie und Sicherheit“ (Frank \& Lorenz, 2015, Deutschland) lenkt den Fokus auf Europa. Der Film zeigt am Beispiel Griechenlands, Spaniens, Irlands und Islands auf, welche Auswirkungen die Bankenkrise auf diese Staaten hatte. Gemeinsame Tendenz ist, dass - und daher wird im Film auch von einem Geschäftsmodell der Banken gesprochen - die Gewinne der Banken an die Aktionäre ausbezahlt werden, während die Verluste durch Regierungen oder die europäische Staatengemeinschaft übernommen und auf die Bevölkerung überwälzt werden. Letztere wird Jahre oder Jahrzehnte sowohl durch Steuererhöhungen als auch durch enorme Kürzungen in den Bereichen Soziales, Wohnen, Bildung und Kultur belastet. Die Begleiteffekte sind Arbeitslosigkeit, Delogierung, Löhne wie in Entwicklungsländern, fehlende Krankenversicherungen und medizinische Versorgung sowie psychische Destabilisierung bis hin zum Suizid, die der Gemeinschaft aufgebürdet werden. Ältere Generationen und Pensionisten müssen die Versorgung ihrer arbeitslosen erwachsenen Kinder übernehmen. Junge Menschen sehen im eigenen Land keine Chancen mehr. Eine Griechin, ehemaliger Mittelstand, bringt es auf den Punkt: „Wir glauben an nichts mehr. Du lässt dein Kind nicht verhungern.“ In Griechenland kostete die Bankenrettung zum Beispiel 50 Milliarden Euro - genauso viel wurde in den oben genannten Bereichen eingespart.

Ein Vertreter der „Occupy Wallstreet“-Bewegung spricht von „Kreditokratie“, einem Modell, das in Amerika längst verwirklicht ist, den Banken Geldflüsse sichert und nun auch in Europa Fuß fasst: Die gesamten Lebenskosten werden über Schulden finanziert, die man über die Jahre hin anhäuft, angefangen von den Ausbildungskosten bis hin zu den Wohnungskosten und den Aufwendungen für die Lebenserhaltung. Mario Draghi, einstiger Goldman-Sachs-Vizepräsident und späterer EZB-Präsident, wird im Film mit folgender Aussage zitiert: „Das europäische Sozialmodell ist Vergangenheit. Die Rettung des Euro wird viel Geld kosten. Das bedeutet, vom europäischen Sozialmodell Abschied zu nehmen." Aber wie konnte es zu diesen Entwicklungen überhaupt kommen?

Im Ländervergleich wird immer wieder derselbe Mechanismus sichtbar, der auf einer Verflechtung von politischen und wirtschaftlichen Entscheidungen basiert: Gesetzesänderungen setzen Deregulierungsprozesse in der Finanzwirtschaft in Gang, die eine „Entfesselung“ des Kapitals zur Folge haben. Dabei spielen Derivate eine große Rolle, das sind Finanzprodukte, die im Wesentlichen

lichen Körper durch Waren zu vervollständigen und ihn zum Warenkörper zu machen" (Decker, 2016, S. 80). 
auf Bündelungen von Kreditforderungen beruhen. Je nach Zusammensetzung können dies Forderungen sein, die von geringem bis hin zu einem hohen Ausfallsrisiko reichen. Kreditausfallsversicherungen, sogenannte SWAPS, ermöglichen den Banken, weit mehr Kredite zu vergeben, als sie aufgrund des vorhandenen Eigenkapitals gewähren dürften. Wenn nun eine Bank wie die Deutsche Bank 96 Prozent des Umsatzes mit Derivaten und Vermögensverwaltung gemacht hat, bedeutet das, dass nur vier Prozent der realen Wirtschaft dienen.

Als positives Beispiel wird im Film Island zitiert, das die Krise zum Anlass nahm, eine Neugestaltung des Finanz- und Wirtschaftssektors vorzunehmen. Unter aktiver Beteiligung der Bevölkerung wurden die sozial Schwächsten geschont, aber alle anderen Bevölkerungsschichten in den Abbau der Schulden einbezogen. Die Vermögen von verschuldeten Banken und Betrieben wurden real bewertet, der spekulative Sektor wurde in Konkurs überführt und die Verfehlungen der Beteiligten wurden durch die Justiz geahndet. Die Ursachen der Krise und ihre Handhabung wurden aufgearbeitet und liegen in mehreren Bänden als „historisches Dokument“ für all jene vor, die sich in der Gegenwart oder der Zukunft damit auseinandersetzen wollen.

Was der Film zeigt, ist, dass über mehr Bürgerbeteiligung, über Wissen, wie Krisen zustande kommen, aber auch durch soziale Gerechtigkeit und eine funktionierende Rechtsprechung Krisenbewältigung möglich ist. Er zeigt daher auch, dass sowohl das Verbergen, Verschieben, Täuschen und Betrügen, dargestellt am Beispiel der Derivate, die metaphorisch als verdinglichte Abwehr von Schuld und Schulden aufgefasst werden können, als auch die Verflochtenheit von Politik und Wirtschaft auf nationaler und internationaler Ebene dazu dienen, die Umverteilung von arm zu reich und von Volkswirtschaften zu Institutionen wie Banken zu beschleunigen.

Die besprochenen Filme geben Auskunft über Daten und Fakten der Finanzund Wirtschaftskrise und die sie begleitenden materiellen und psychischen Folgen, geprägt von der Kunstform des Films und der Dokumentation.

Auf dem Kongress „Moments of Crisis“ der New Lacanian School of Psychoanalysis im Mai 2015 in Genf wurde ein Ausschnitt aus dem Spielfilm „Sonntags ... nie!“ (Dassin, 1960, Frankreich) mit Melina Mercouri gezeigt: In einer gut besuchten Taverne tanzt ein Mann allein inmitten des Raumes und trinkt, lässt die Gläser zerschellen, was mit dem Klingeln der Registrierkasse verbucht wird. Schönheit, Trauer, Erotik und Aggression verbinden und entfalten sich. So gesehen gibt es kein Genießen, ohne dass man dafür bezahlen muss. 
Open Access Dieses Kapitel wird unter der Creative Commons Namensnennung 4.0 International Lizenz (http://creativecommons.org/licenses/by/4.0/deed.de) veröffentlicht, welche die Nutzung, Vervielfältigung, Bearbeitung, Verbreitung und Wiedergabe in jeglichem Medium und Format erlaubt, sofern Sie den/die ursprünglichen Autor(en) und die Quelle ordnungsgemäß nennen, einen Link zur Creative Commons Lizenz beifügen und angeben, ob Änderungen vorgenommen wurden.

Die in diesem Kapitel enthaltenen Bilder und sonstiges Drittmaterial unterliegen ebenfalls der genannten Creative Commons Lizenz, sofern sich aus der Abbildungslegende nichts anderes ergibt. Sofern das betreffende Material nicht unter der genannten Creative Commons Lizenz steht und die betreffende Handlung nicht nach gesetzlichen Vorschriften erlaubt ist, ist für die oben aufgeführten Weiterverwendungen des Materials die Einwilligung des jeweiligen Rechteinhabers einzuholen.

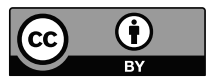

